\title{
Solubility Determination and Correlation of Warfarin Sodium 2-Propanol Solvate in Pure, Binary, and Ternary Solvent Mixtures
}

Mery Vet George De la Rosa, ${ }^{12}$ Roberto Santiago, ${ }^{2,3}$ Joseph Malavé Romero, ${ }^{2,4}$ Jorge Duconge,' Jean-Christophe Monbaliu, ${ }^{5}$ Vilmalí López-Mejías, ${ }^{2,6 *}$ and Torsten Stelzer ${ }^{2 *}$

'Department of Pharmaceutical Sciences, University of Puerto Rico - Medical Sciences Campus, San Juan, Puerto Rico, 00936, United States

${ }^{2}$ Crystallization Design Institute, Molecular Sciences Research Center, University of Puerto Rico, San Juan, PR 00926, United States

${ }^{3}$ Department of Mathematics, University of Puerto Rico - Río Piedras Campus, San Juan, Puerto Rico, 00931, United States

${ }^{4}$ Department of Biology, University of Puerto Rico - Bayamón Campus, Bayamón, Puerto Rico, 00959, United States

Center for Integrated Technology and Organic Synthesis, RU MolSys, University of Liège, B-4000 Liège (Sart Tilman), Belgium

'Department of Chemistry, University of Puerto Rico - Río Piedras Campus, San Juan, Puerto Rico, 00931, United States

*E-mail: torsten.stelzer@upr.edu (T.S.)

*E-mail: vilmali.lopez@upr.edu (V.L.-M.) 


\section{Differential Scanning Calorimetry (DSC) \& Thermogravimetric Analysis (TGA)}

The melting temperature $T_{m}$ and enthalpy of fusion $\Delta_{f u s} H$ of warfarin sodium (WS) were determined by DSC Q2000 (TA Instruments Inc.) equipped with a RCS40 single-stage refrigeration system. The calibration of the instrument was performed with an indium standard $\left(T_{m}=429.75 \mathrm{~K}\right.$ and $\left.\Delta_{f u s} H=28.54 \mathrm{~J} / \mathrm{g}\right)$. Samples $(\sim 2.200 \mathrm{mg})$ were weighed using a microbalance (XP26, Mettler Toledo, uncertainty $\pm 0.002 \mathrm{mg}$ ) and placed on sealed pans with a pinhole, the preferred method when studying solvates. ${ }^{-4}$ Samples were equilibrated at $298.15 \mathrm{~K}$ for 10 min prior to heating to $523.15 \mathrm{~K}$ under a $\mathrm{N}_{2}$ atmosphere $(50 \mathrm{~mL} / \mathrm{min})$ at a rate of 10.0 $\mathrm{K} / \mathrm{min}$ and a temperature accuracy of $0.1 \mathrm{~K}$. Thermograms were analyzed using the software $T A$ Universal Analysis 2000 (version 4.5A). The measurements were conducted five times $(n=5)$ and the average result of the peak temperature was taken to ensure accuracy (Table S1). Representative DSC and TGA thermographs of WS - IPA are shown in Figure S1. The area under the curve represents the enthalpy of fusion, in $\mathrm{J}^{-\mathrm{g}^{-1}}$. To obtain the value in $\mathrm{kJ} \cdot \mathrm{mol}^{-1}$, the enthalpy of fusion in $\mathrm{J}^{\mathrm{g}} \mathrm{g}^{-1}$ was multiplied by the molecular mass of WS (330.31 $\left.\mathrm{g} \cdot \mathrm{mol}^{-1}\right)$ and divided by 1,000 . The average $\Delta_{f u s} H$, and $T_{m}$ obtained for WS were determined as $71 \pm 2 \mathrm{~kJ} / \mathrm{mol}^{-1}$ and $468.87 \pm 0.05 \mathrm{~K}$, respectively. The average value of $T_{m}$ was employed to calculate the correlated mole fraction solubility $\left(x_{1}^{c a l}\right)$ using the $\lambda \mathrm{h}$ model equation. The standard uncertainty $u$ for the experimental temperature measured with DSC was estimated to be $u\left(T_{m}\right)=0.5 \mathrm{~K}$.

The desolvation and degradation of WS - IPA were recorded in a TGA Q500 (TA Instruments Inc.) calibrated with calcium oxalate monohydrate. Samples (5-10 mg) were equilibrated at $298.15 \mathrm{~K}$ for $10 \mathrm{~min}$ prior to heating to $523.15 \mathrm{~K}$ under $\mathrm{N}_{2}$ atmosphere $(60$ $\mathrm{mL} / \mathrm{min}$ ) at a rate of $5.0 \mathrm{~K} / \mathrm{min}$ and a temperature accuracy of $0.1 \mathrm{~K}$. Data was analyzed with TA Universal Analysis software v 4.5A. A solvent loss of about $\sim 8 \%$ was observed prior to the melting of the desolvated WS (Figure S1 bottom). 
Table S1. Thermodynamics properties of WS at a pressure $(p), p=101.3 \mathrm{kPa}$.

$\begin{array}{rcc} & T_{\mathrm{m}, \text { peas }} / \mathrm{K} & \Delta_{\text {fus }} H / \mathrm{kJ} \cdot \mathrm{mol}^{-1} \\ & 468.91 & 71.15 \\ & 468.78 & 69.81 \\ & 468.92 & 69.45 \\ & 468.85 & 73.82 \\ & 468.88 & 71.16 \\ \text { Average (n = 5) } & 468.87 & 71.08 \\ S T D & 0.05 & 1.72\end{array}$

standard uncertainty $u$ is $u(T)=0.5 \mathrm{~K}$. Relative standard uncertainty $u_{\mathrm{s}}$ is $u_{i}(p)=0.1$.
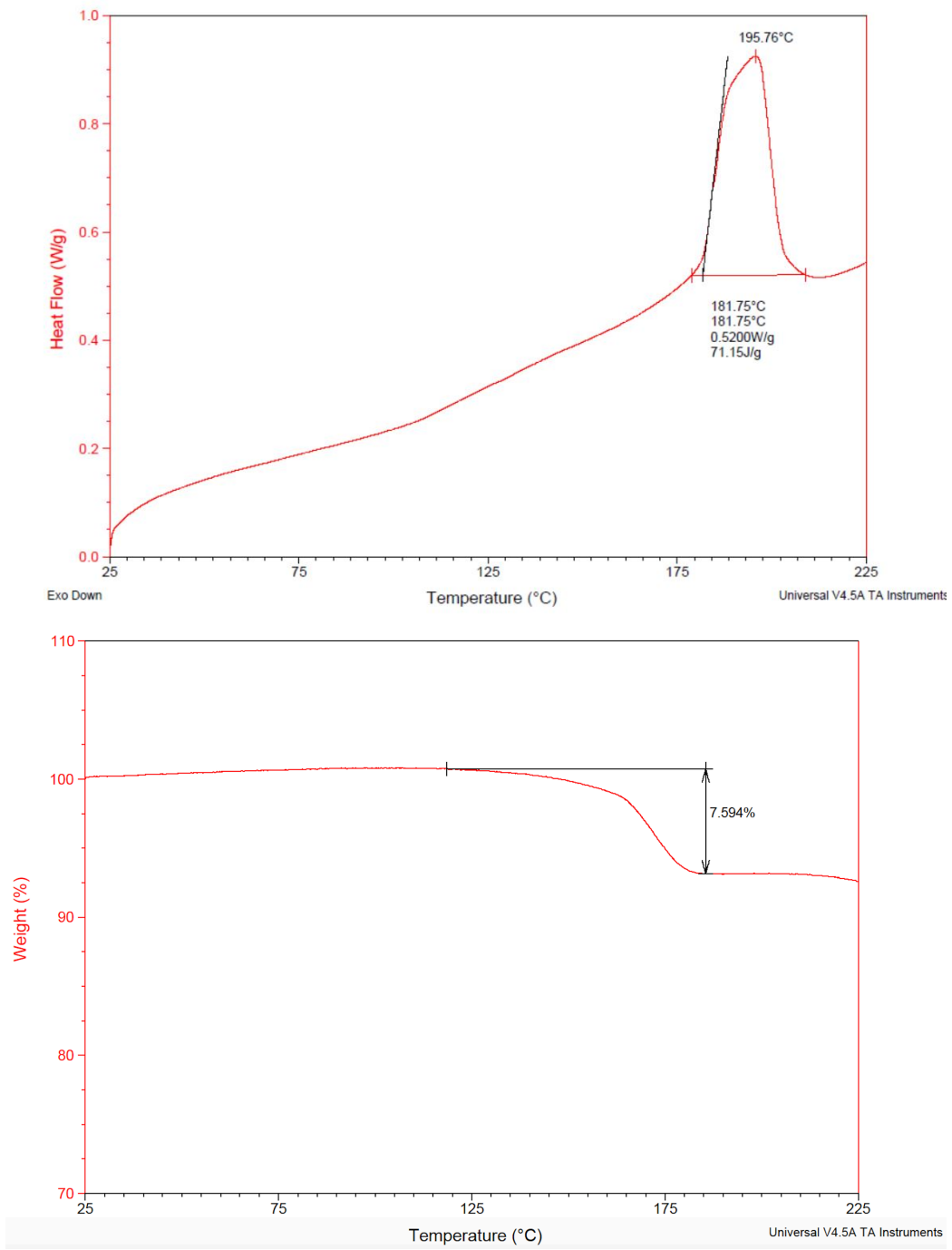

Figure S1. Representative thermograms of warfarin sodium isopropanol solvate (WS • IPA), (top) DSC and (bottom) TGA.

The peak melting temperature $\left(T_{m}\right)$ for WS, was reported previously by Gao et al. ${ }^{(460.15}$ $\mathrm{K}$ ) using an open pan, but was re-determined experimentally within this study using hermetically sealed pans with a pinhole, the preferred method when studying solvates..$^{24.7}$ The $T_{m}$ of WS 
obtained within this work $(468.87 \mathrm{~K})$ was used to calculate the mole fraction solubility $\left(x_{1}^{c a l}\right)$ applying the $\lambda \mathrm{h}$ model equation.

\section{Solubility Measurements}

In this study the solubility of WS • IPA was determined applying the polythermal method ${ }^{8-11}$ using a Crystal16 multiple reactor system (Technobis Crystallization Systems). ${ }^{9,0,12-14}$ Sealed glass vials with an internal volume of $2 \mathrm{~mL}$ (Fisher Scientific) were employed to prepare the samples with different concentrations using a microbalance (XP26, Mettler Toledo, uncertainty \pm 0.002 $\mathrm{mg}$ ) to weigh the solute and an analytical balance (MS104S, Mettler Toledo, uncertainty \pm 0.1 $\mathrm{mg}$ ) to weigh the pure solvents and solvent mixtures. The resulting suspensions were agitated using a magnetic stir bar (rare earth) at $700 \mathrm{rpm}$ while heated from 278.15 to $333.15 \mathrm{~K}$ at 0.3 $\mathrm{K} / \mathrm{min} .{ }^{14}$ Due to the boiling point restriction of acetone $(329.15 \mathrm{~K}){ }^{15}$ the temperature range needed to be adjusted to 278.15 to $323.15 \mathrm{~K}$ for the pure solvent and all solvent mixtures containing this solvent. Monitoring the transmission of light through the suspension was used to determine the saturation temperature at its maximum, under the assumption that dissolution kinetics can be neglected. To ensure accuracy, each concentration was measured at least in duplicate. The uncertainty of each saturated temperature was within $\pm 0.1 \mathrm{~K}$.

\subsection{Surface Plots of Solubility with $\lambda$ h Equation}

Origin (OriginLab Corporation, version B95.0.193) was employed to generate the surface plots using the Random Kriging Corelation method for $x y z$ gridding. The number of columns and rows for the gridding were chosen based on the maximum number of data points and number of different solvent compositions observed within each particular system of solvent mixtures. The search radius and smoothing were set to two and one, respectively, while the minimum and maximum points were set to 10 and 100, respectively. Figures S2-S11, present the surface plots of the calculated solubility data of WS - IPA in binary and ternary solvent mixtures using the $\lambda$ $\mathrm{h}$ model equation. 


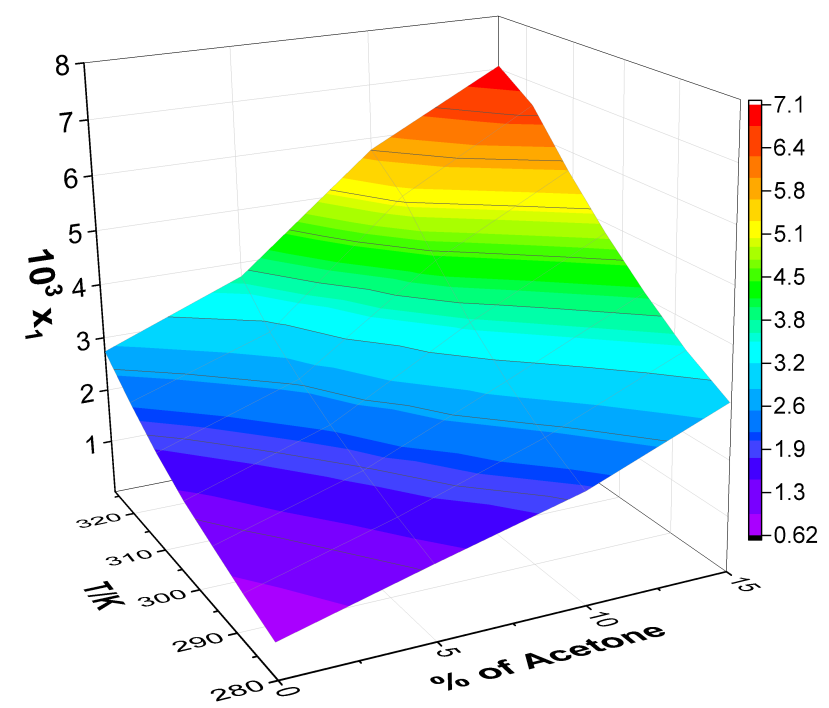

Figure S2. Surface plot for the solubility of WS • IPA (1) in binary solvent system isopropanol (2) + acetone (3) correlated with the $\lambda \mathrm{h}$ equation. $x_{1}$ represents the mole fraction solubility of WS - IPA and $T$ the temperature in kelvin (K).

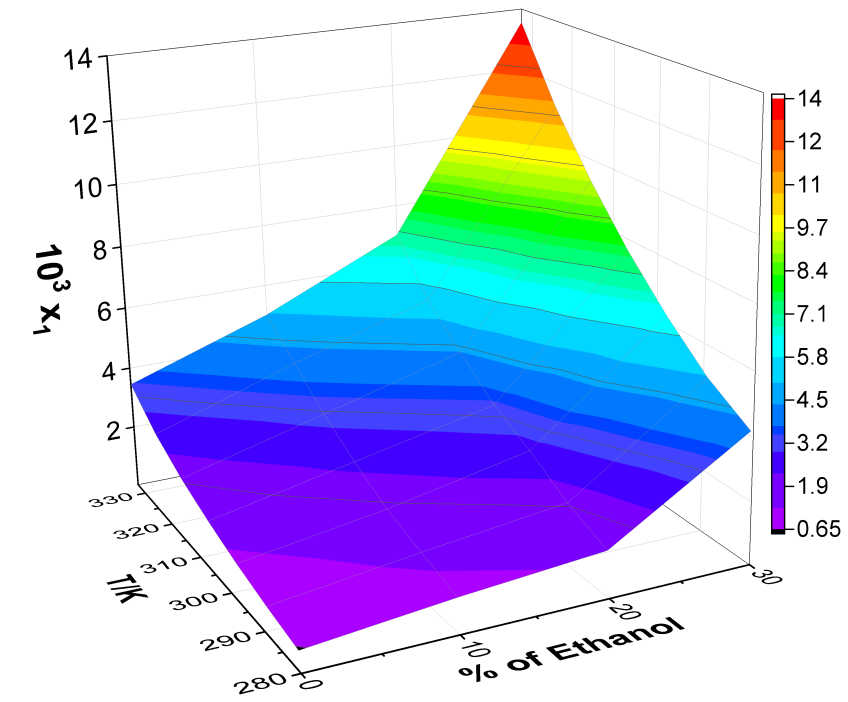

Figure S3. Surface plot for the solubility of WS • IPA (1) in binary solvent system isopropanol (2) + ethanol (3) correlated with the $\lambda \mathrm{h}$ equation. $x_{1}$ represents the mole fraction solubility of WS $\bullet$ IPA and $T$ the temperature in kelvin (K). 


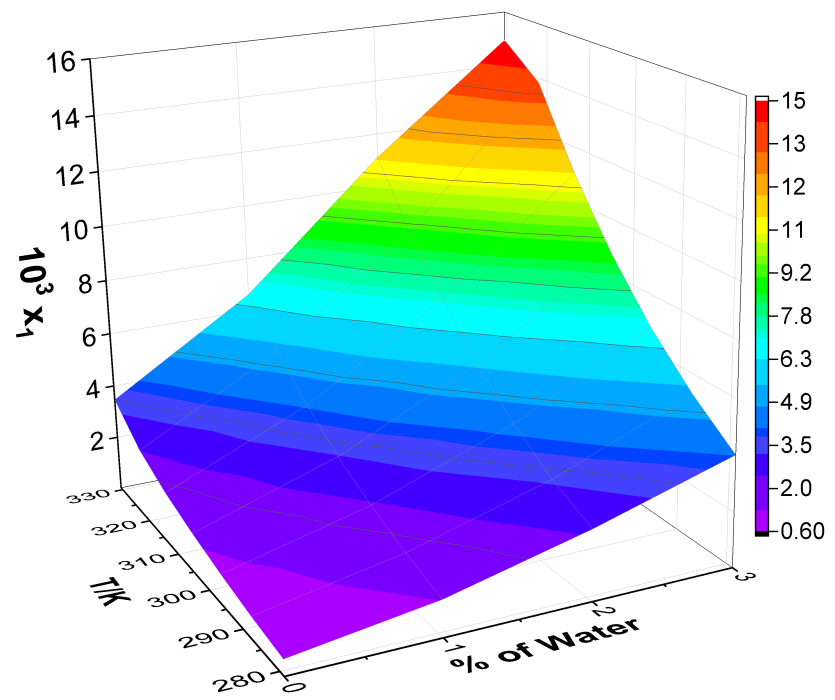

Figure S4. Surface plot for the solubility of WS • IPA (1) in binary solvent system isopropanol (2) + water (3) correlated with the $\lambda$ h equation. $x_{1}$ represents the mole fraction solubility of WS $\bullet$ IPA and $T$ the temperature in kelvin $(\mathrm{K})$.

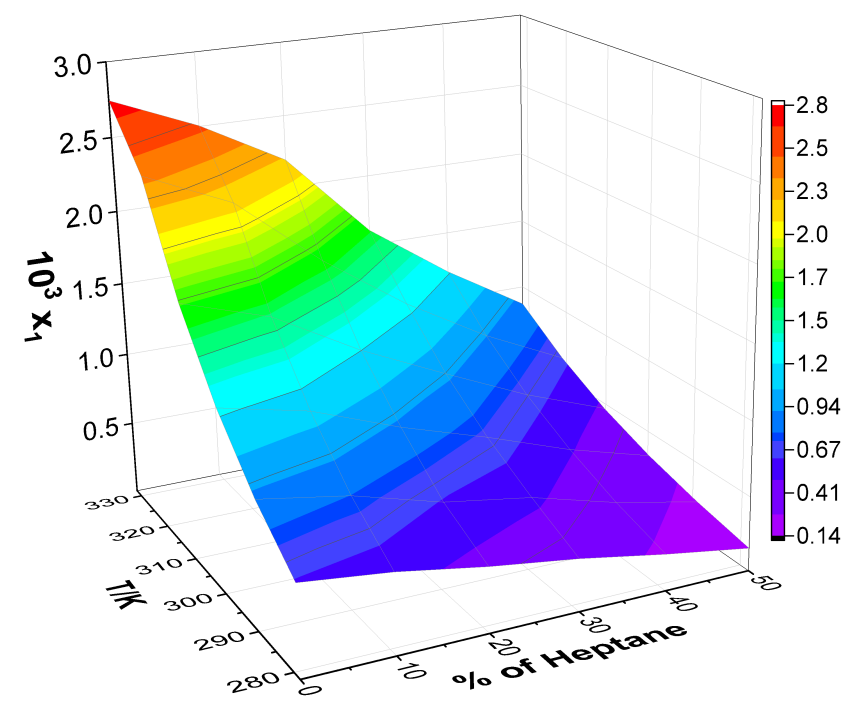

Figure S5. Surface plot for the solubility of WS • IPA (1) in binary solvent system isopropanol (2) + heptane (3) correlated with the $\lambda \mathrm{h}$ equation. $x_{1}$ represents the mole fraction solubility of WS $\bullet$ IPA and $T$ the temperature in kelvin $(\mathrm{K})$. 


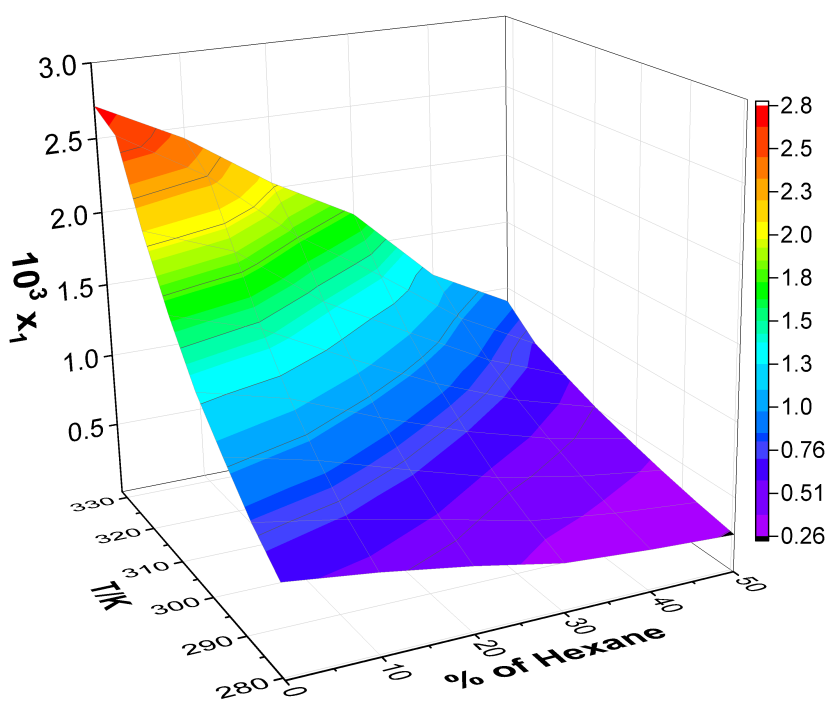

Figure S6. Surface plot for the solubility of WS • IPA (1) in binary solvent system isopropanol (2) + hexane (3) correlated with the $\lambda$ h equation. $x_{1}$ represents the mole fraction solubility of WS - IPA and $T$ the temperature in kelvin (K).

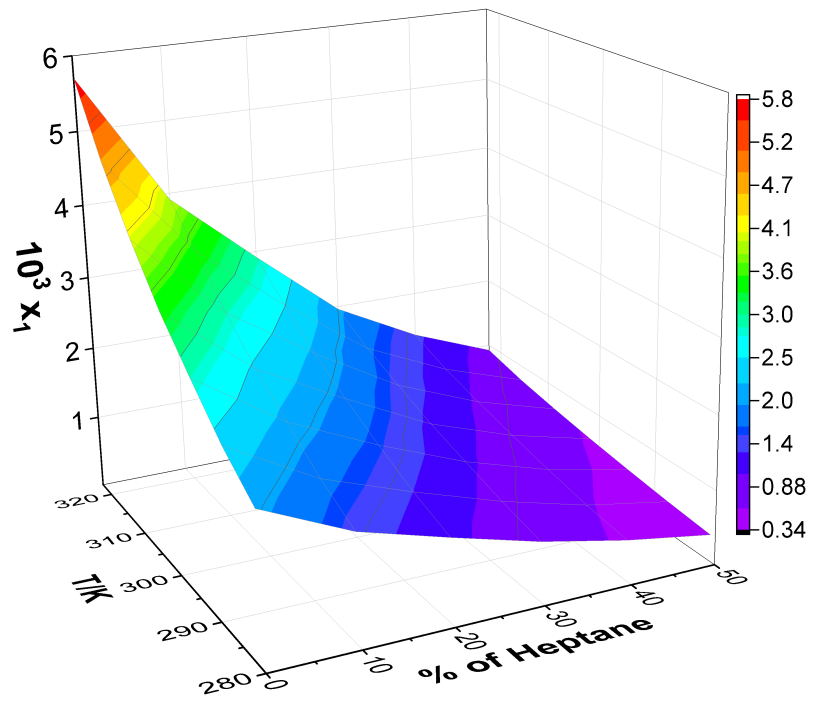

Figure S7. Surface plot for the solubility of WS - IPA (1) in ternary solvent system isopropanol (2) + acetone (3) with $W_{3}=0.10+$ heptane (4) correlated with the $\lambda$ h equation. $x_{1}$ represents the mole fraction solubility of WS - IPA and $T$ the temperature in kelvin (K). 


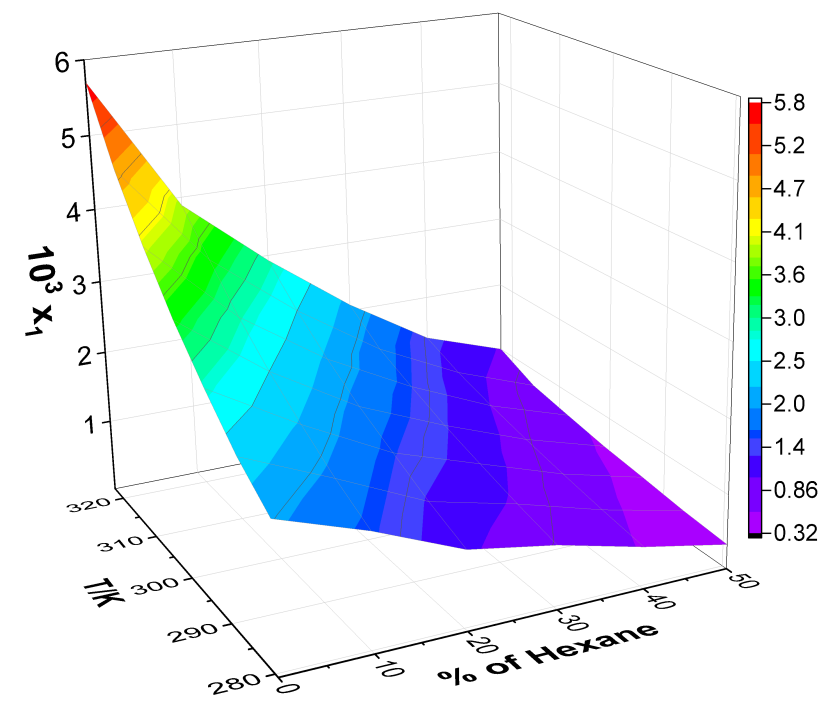

Figure S8. Surface plot for the solubility of WS - IPA (1) in ternary solvent system isopropanol (2) + acetone (3) with $W_{3}=0.10+$ hexane (4) correlated with the $\lambda \mathrm{h}$ equation. $x_{1}$ represents the mole fraction solubility of WS - IPA and $T$ the temperature in kelvin (K).

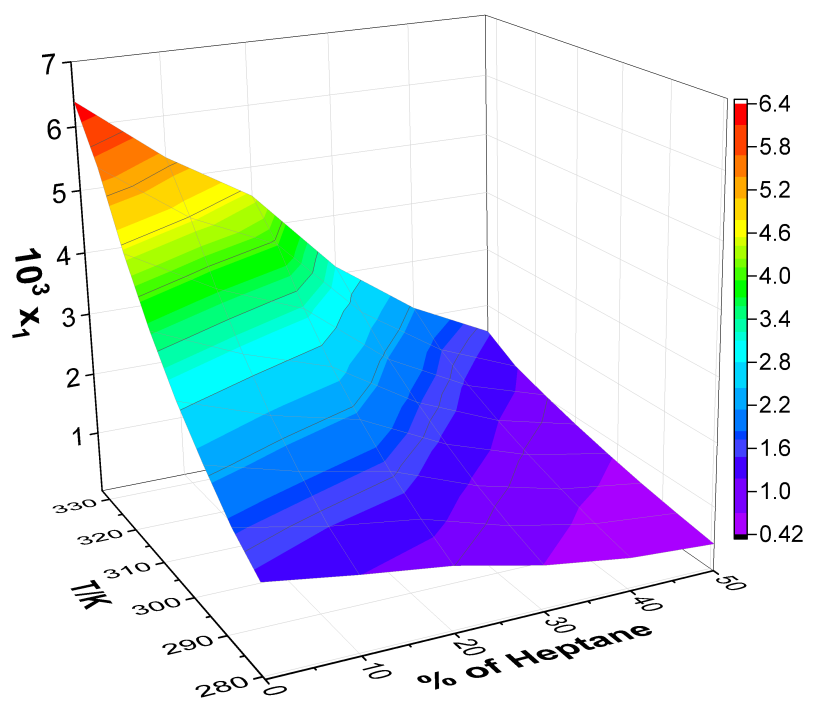

Figure S9. Surface plot for the solubility of WS - IPA (1) in ternary solvent system isopropanol (2) + ethanol (3) with $W_{3}=0.20+$ heptane (4) correlated with the $\lambda$ h equation. $x_{1}$ represents the mole fraction solubility of WS - IPA and $T$ the temperature in kelvin (K). 


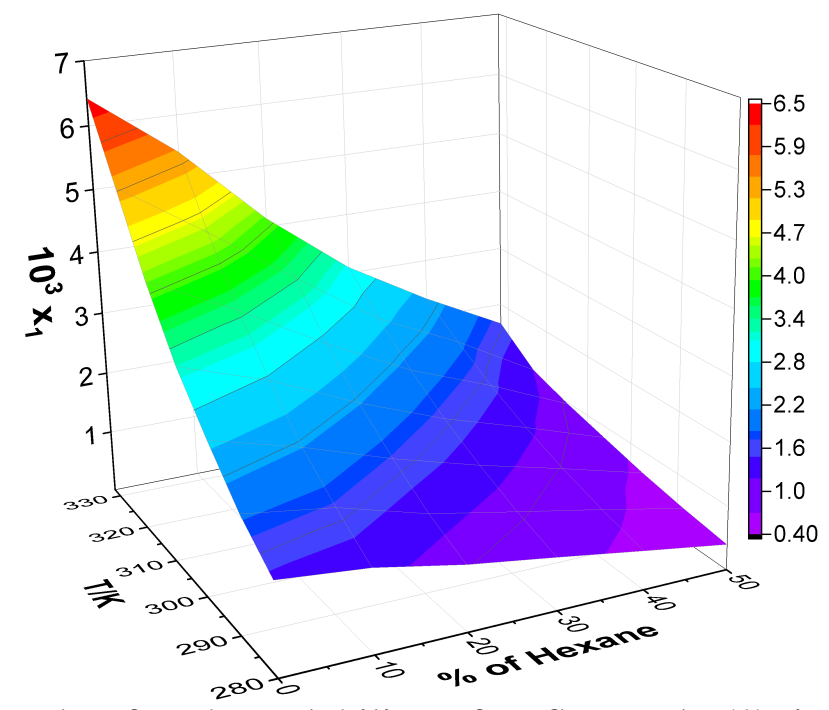

Figure S10. Surface plot for the solubility of WS - IPA (1) in ternary solvent system isopropanol (2) + ethanol (3) with $W_{3}=0.20+$ hexane (4) correlated with the $\lambda \mathrm{h}$ equation. $x_{1}$ represents the mole fraction solubility of WS - IPA and $T$ the temperature in kelvin (K).

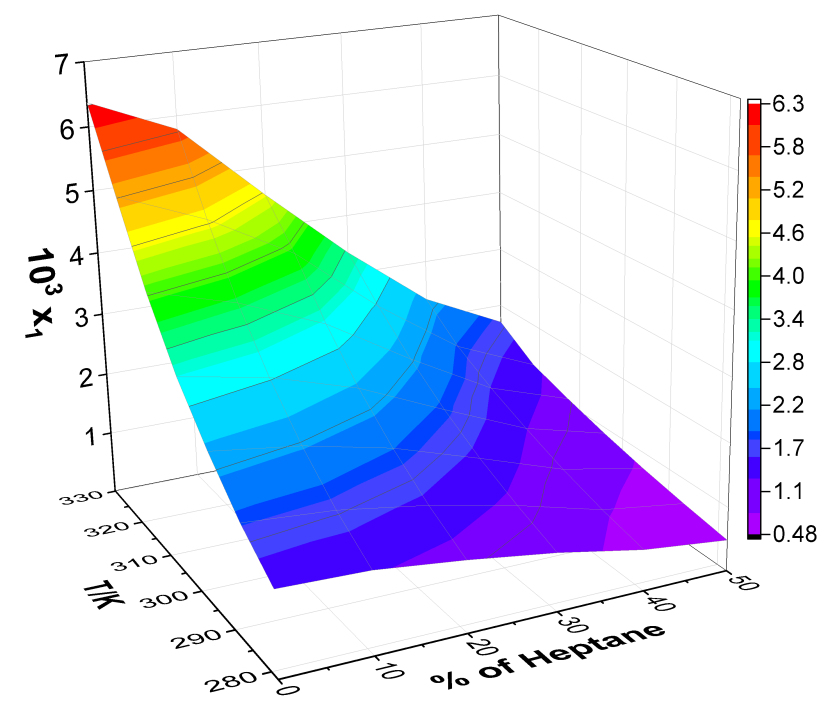

Figure S11. Surface plot for the solubility of WS - IPA (1) in ternary solvent system isopropanol (2) + water (3) with $W_{3}=0.01+$ heptane (4) correlated with the $\lambda$ h equation. $x_{1}$ represents the mole fraction solubility of WS - IPA and $T$ the temperature in kelvin (K).

\subsection{Scatter Plots for Solubility}

Figure S12 shows the calculated and experimentally derived solubility data (scatter plot) of WS - IPA in the pure solvents using the $\lambda \mathrm{h}$ model equation. Figures S13-22 depict the calculated and experimentally derived solubility data (scatter plot) for WS - IPA using the modified Apelblat equation (left side) and the $\lambda \mathrm{h}$ model equation (right side) in each of the solvent mixtures. 


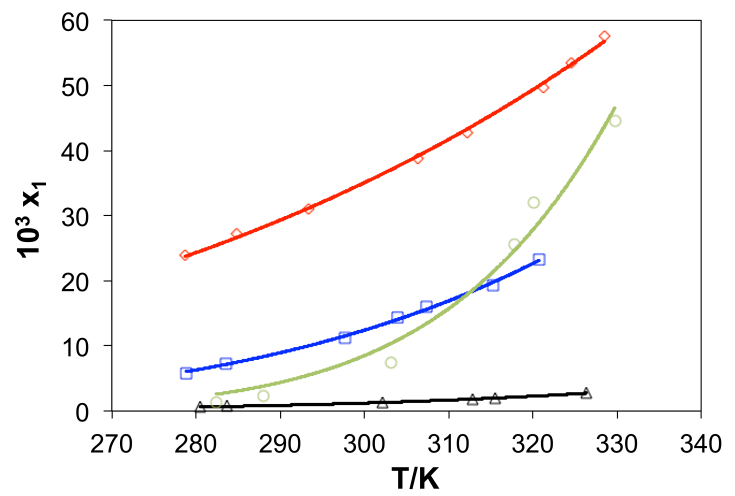

Figure S12. Experimental and correlated solubility data of WS - IPA in pure solvents, from top to bottom; $\diamond$, ethanol; $\square$, acetone; $\odot$, water; $\Delta$, isopropanol; - , calculated using the $\lambda \mathrm{h}$ equation. $x_{1}$ represents the mole fraction solubility of WS $\bullet$ IPA and $T$ the temperature in kelvin (K).
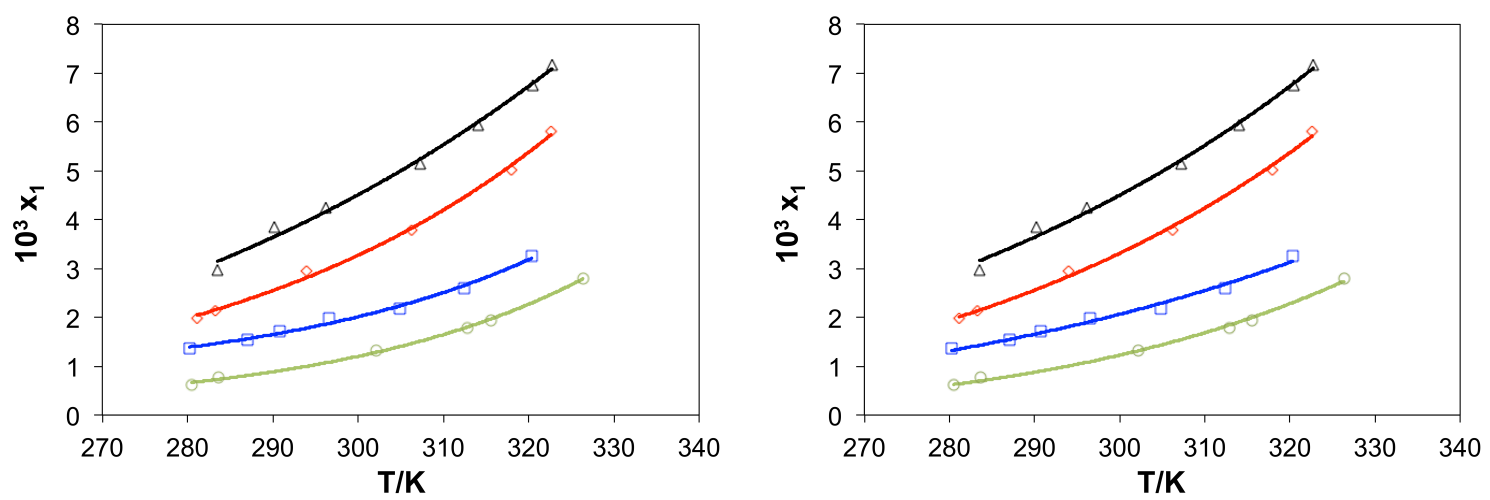

Figure S13. Experimental and correlated solubility data of WS - IPA (1) in binary isopropanol (2) + acetone (3) solvent mixtures. $\circ, W_{3}=0$ (isopropanol); $\square, W_{3}=0.05 ; \diamond, W_{3}=0.11 ; \Delta, W_{3}=$ 0.15 ; - calculated using the modified Apelblat equation (left) and $\lambda \mathrm{h}$ equation (right). $W_{3}$ is the mass fraction of acetone (3) in binary isopropanol (2) + acetone (3) mixture. $x_{1}$ represents the mole fraction solubility of WS • IPA and $T$ the temperature in kelvin (K). 

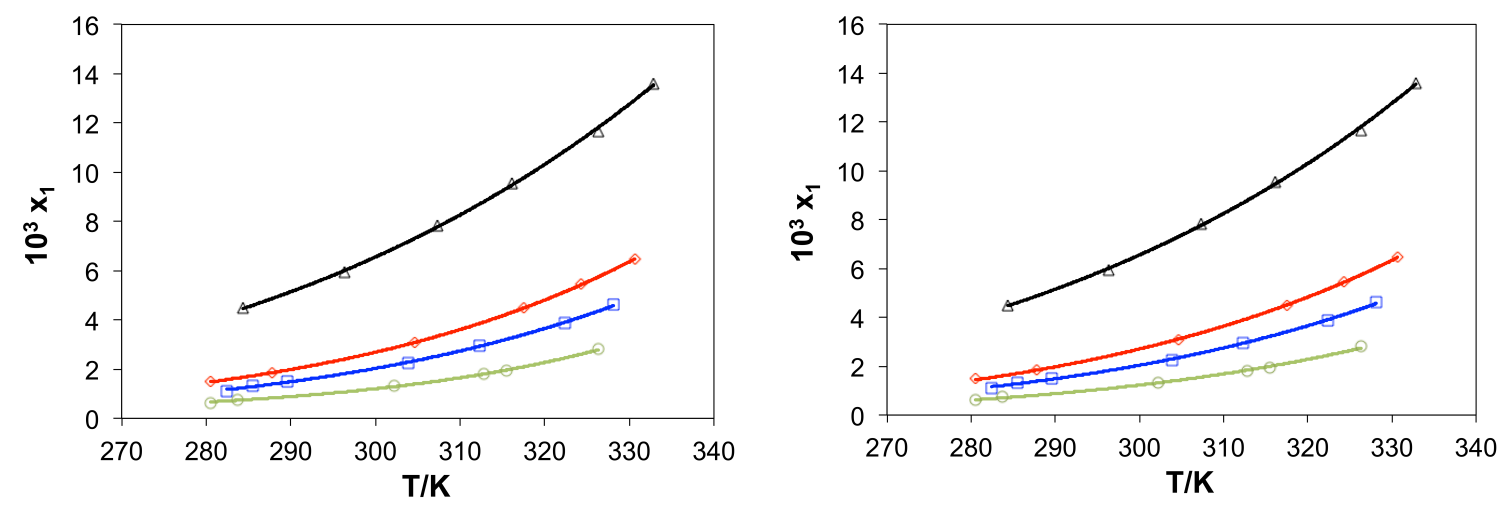

Figure S14. Experimental and correlated solubility data of WS - IPA (1) in binary isopropanol (2) + ethanol (3) solvent mixtures. $\circ, W_{3}=0$ (isopropanol); $\square, W_{3}=0.10 ; \diamond, W_{3}=0.20 ; \Delta, W_{3}=$ $0.30 ;-$, calculated using the modified Apelblat equation (left) and $\lambda \mathrm{h}$ equation (right). $W_{3}$ is the mass fraction of ethanol (3) in binary isopropanol (2) + ethanol (3) mixture. $x_{1}$ represents the mole fraction solubility of WS • IPA and $T$ the temperature in kelvin $(\mathrm{K})$.
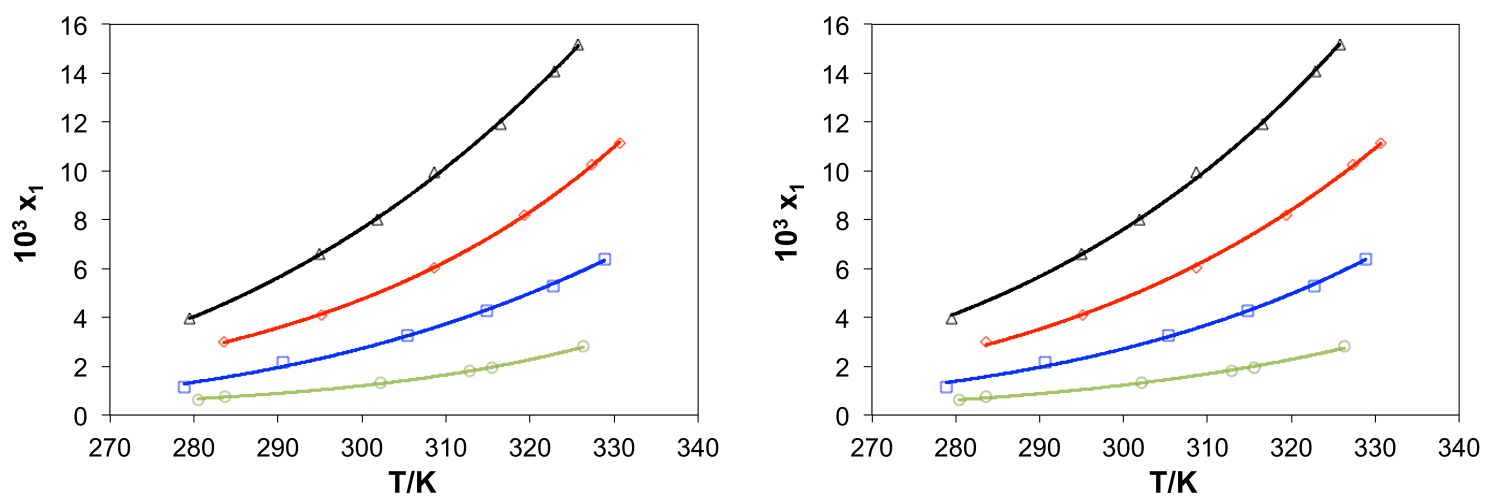

Figure S15. Experimental and correlated solubility data of WS $\bullet$ IPA (1) in binary isopropanol (2) + water (3) solvent mixtures. $\bigcirc, W_{3}=0$ (isopropanol); $\square, W_{3}=0.01 ; \diamond, W_{3}=0.03 ; \Delta, W_{3}=0.04$; - , calculated using the modified Apelblat equation (left) and $\lambda \mathrm{h}$ equation (right). $W_{3}$ is the mass fraction of water (3) in binary isopropanol (2) + water (3) mixture. $x_{1}$ represents the mole fraction solubility of WS • IPA and $T$ the temperature in kelvin $(\mathrm{K})$. 

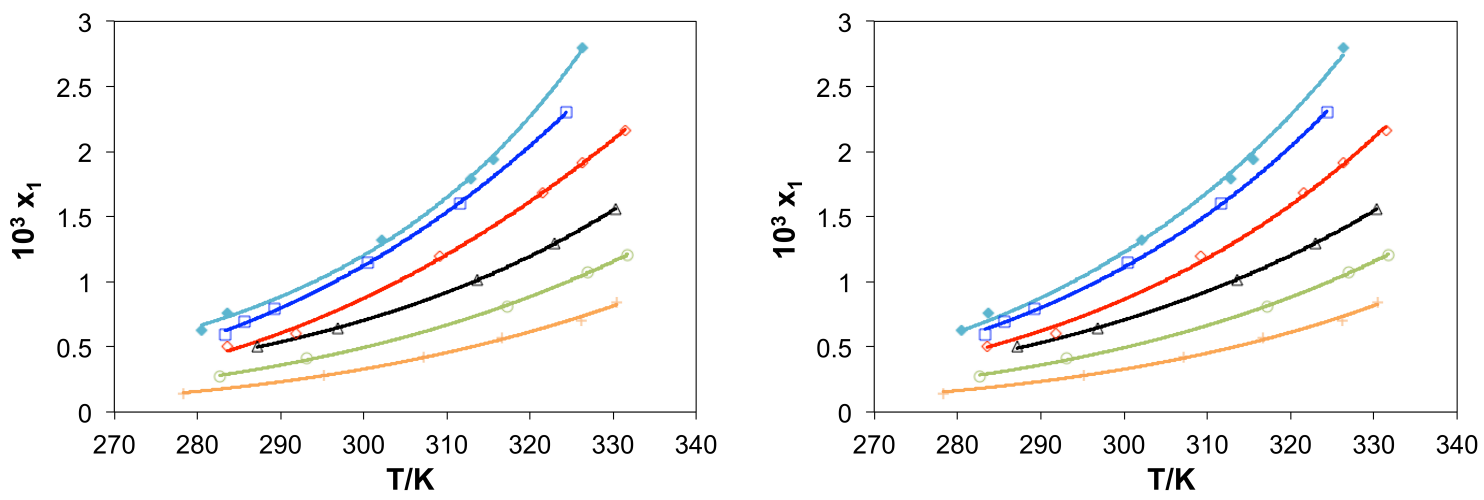

Figure S16. Experimental and correlated solubility data of WS - IPA (1) in binary isopropanol (2) + heptane (3) solvent mixtures. $\bullet, W_{3}=0$ (isopropanol); $\square, W_{3}=0.09 ; \diamond, W_{3}=0.18 ; \Delta, W_{3}=$ $0.28 ; \circ, W_{3}=0.37 ;+, W_{3}=0.47 ;-$, calculated using the modified Apelblat equation (left) and $\lambda$ $\mathrm{h}$ equation (right). $W_{3}$ is the mass fraction of heptane (3) in binary isopropanol (2) + heptane (3) mixture. $x_{1}$ represents the mole fraction solubility of WS - IPA and $T$ the temperature in kelvin $(\mathrm{K})$
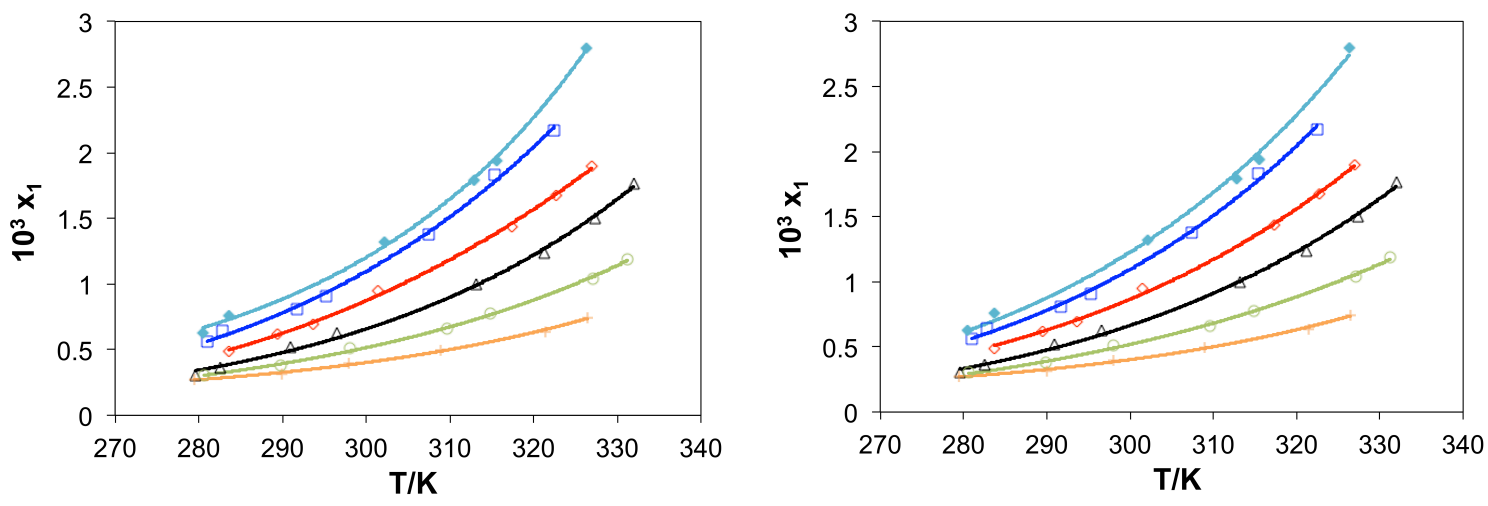

Figure S17. Experimental and correlated solubility data of WS - IPA (1) in binary isopropanol (2) + hexane (3) solvent mixtures. $\bullet, W_{3}=0$ (isopropanol); $\square, W_{3}=0.09 ; \diamond, W_{3}=0.17 ; \Delta, W_{3}=$ $0.27 ; \circ, w_{3}=0.36 ;+, w_{3}=0.46 ;-$, calculated using the modified Apelblat equation (left) and $\lambda$ $\mathrm{h}$ equation (right). $W_{3}$ is the mass fraction of hexane (3) in binary isopropanol (2) + hexane (3) mixture. $x_{1}$ represents the mole fraction solubility of WS - IPA and $T$ the temperature in kelvin (K). 

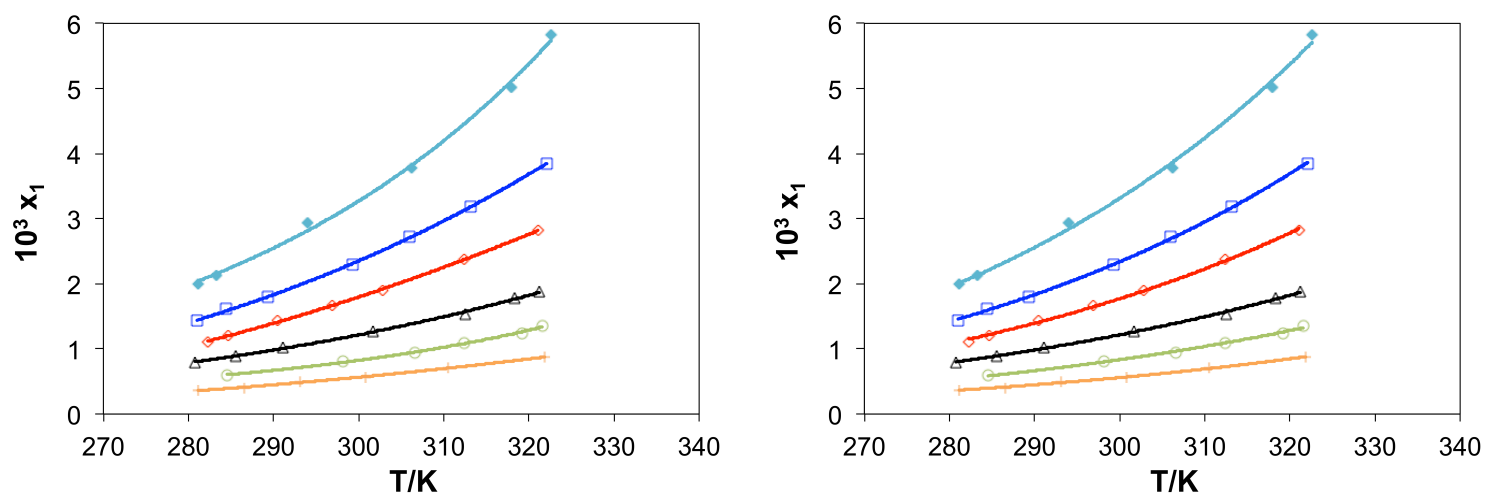

Figure S18. Experimental and correlated solubility data of WS - IPA (1) in ternary isopropanol (2) + acetone (3) with $W_{3}=0.10+$ heptane (4) solvent mixture. $\bullet, W_{4}=0$ (isopropanol + acetone $W_{3}=0.1$ ); $\square, W_{4}=0.09 ; \diamond, W_{4}=0.18 ; \Delta, W_{4}=0.27 ; \circ, W_{4}=0.37 ;+, W_{4}=$ 0.47 ; - calculated using the modified Apelblat equation (left) and $\lambda \mathrm{h}$ equation (right). $W_{3}$ is the mass fraction of acetone (3) in binary isopropanol (2) + acetone (3) mixture. $W_{4}$ is the mass fraction of heptane (4) in ternary isopropanol (2) + acetone (3) + heptane (4) mixture. $x_{1}$ represents the mole fraction solubility of WS - IPA and $T$ the temperature in kelvin (K).
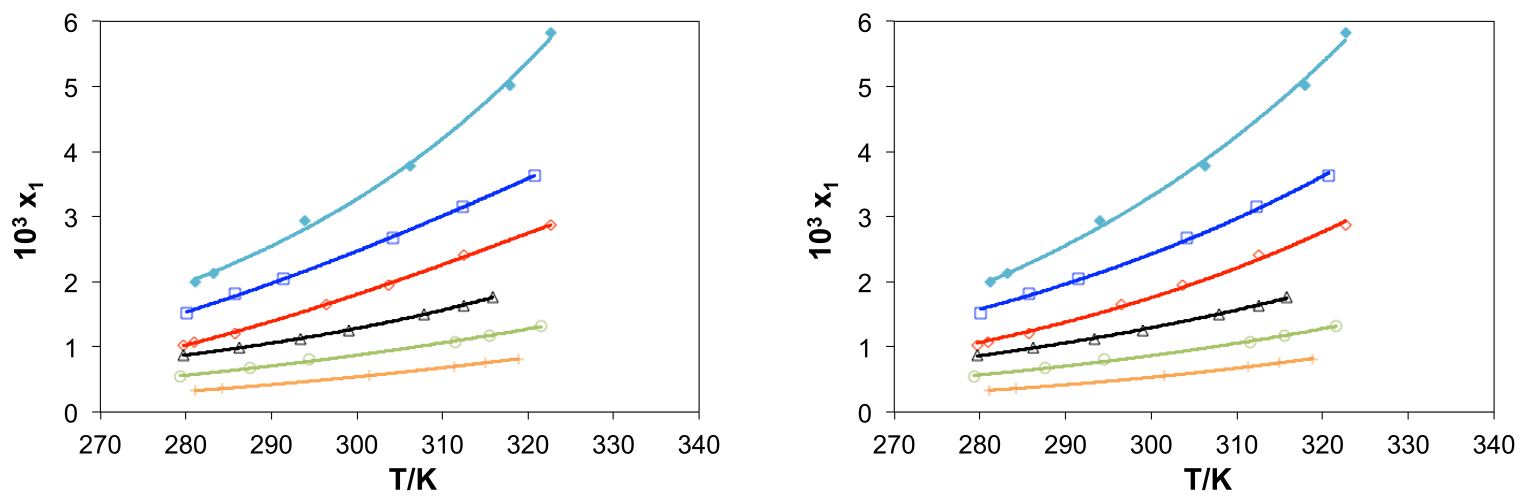

Figure S19. Experimental and correlated solubility data of WS - IPA (1) in ternary isopropanol (2) + acetone (3) with $W_{3}=0.10+$ hexane (4) solvent mixture. $\bullet, W_{4}=0$ (isopropanol + acetone $\left.W_{3}=0.1\right) ; \square, W_{4}=0.09 ; \diamond, W_{4}=0.17 ; \Delta, W_{4}=0.27 ; \circ, W_{4}=0.37 ;+, W_{4}=0.47 ;-$, calculated using the modified Apelblat equation (left) and $\lambda \mathrm{h}$ equation (right). $W_{3}$ is the mass fraction of acetone (3) in binary isopropanol (2) + acetone (3) mixture. $W_{4}$ is the mass fraction of hexane (4) in ternary isopropanol (2) + acetone (3) + hexane (4) mixture. $x_{1}$ represents the mole fraction solubility of WS • IPA and $T$ the temperature in kelvin (K). 

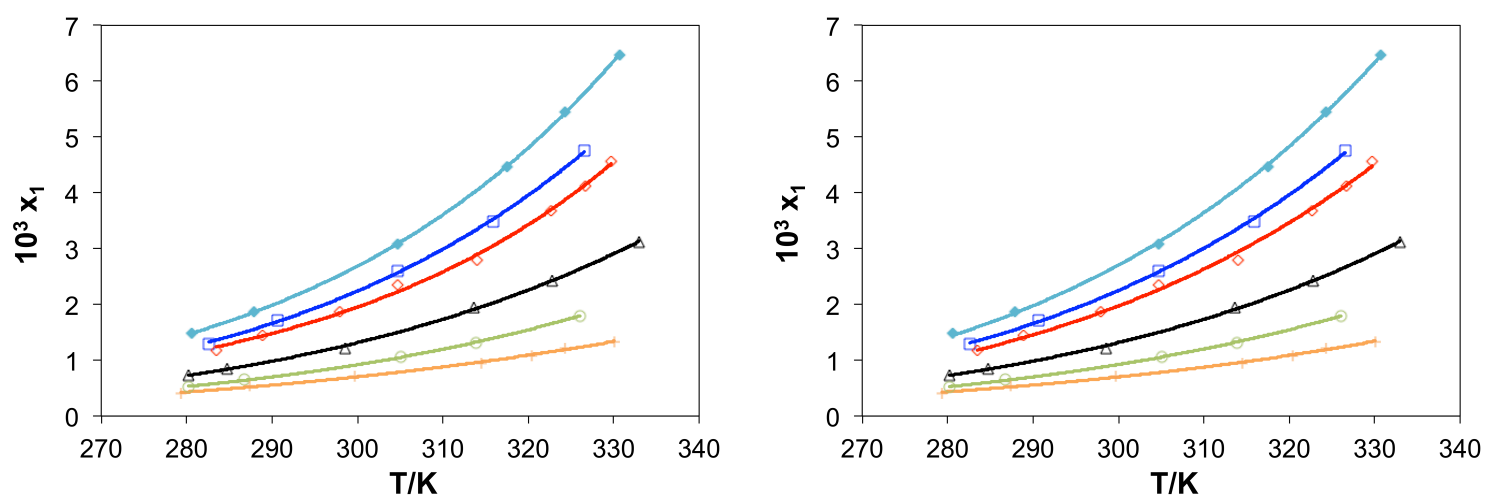

Figure S20. Experimental and correlated solubility data of WS - IPA (1) in ternary isopropanol (2) + ethanol (3) with $W_{3}=0.20+$ heptane (4) solvent mixture. $\bullet, W_{4}=0$ (isopropanol + ethanol $\left.W_{3}=0.20\right) ; \square, W_{4}=0.09 ; \diamond, W_{4}=0.18 ; \Delta, W_{4}=0.27 ; \circ, W_{4}=0.38 ;+, W_{4}=0.47 ;-$, calculated using the modified Apelblat equation (left) and $\lambda \mathrm{h}$ equation (right). $W_{3}$ is the mass fraction of ethanol (3) in binary isopropanol (2) + ethanol (3) mixture. $W_{4}$ is the mass fraction of heptane (4) in ternary isopropanol (2) + ethanol (3) + heptane (4) mixture. $x_{1}$ represents the mole fraction solubility of WS $\bullet$ IPA and $T$ the temperature in kelvin $(\mathrm{K})$.
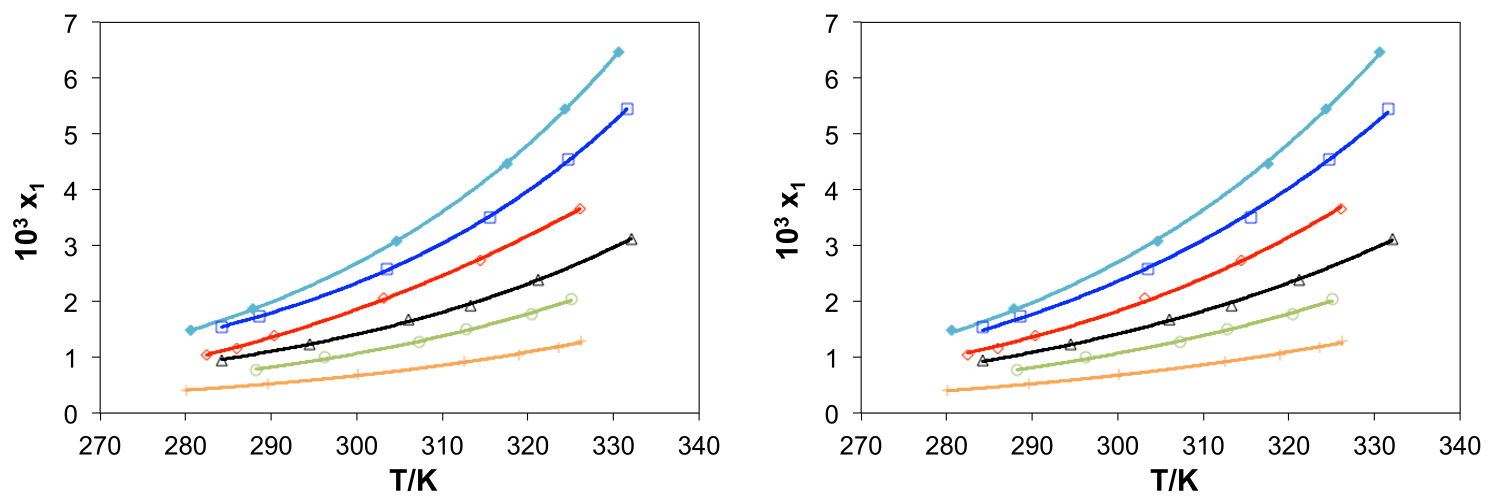

Figure S21. Experimental and correlated solubility data of WS - IPA (1) in ternary isopropanol (2) + ethanol (3) with $W_{3}=0.20+$ hexane (4) solvent mixture. $\bullet, W_{4}=0$ (isopropanol + ethanol $\left.W_{3}=0.20\right) ; \square, W_{4}=0.08 ; \diamond, W_{4}=0.17 ; \Delta, W_{4}=0.27 ; \circ, W_{4}=0.36 ;+, W_{4}=0.46 ;-$, calculated using the modified Apelblat equation (left) and $\lambda \mathrm{h}$ equation (right). $W_{3}$ is the mass fraction of ethanol (3) in binary isopropanol (2) + ethanol (3) mixture. $W_{4}$ is the mass fraction of hexane (4) in ternary isopropanol (2) + ethanol (3) + hexane (4) mixture. $x_{1}$ represents the mole fraction solubility of WS • IPA and $T$ the temperature in kelvin $(\mathrm{K})$. 

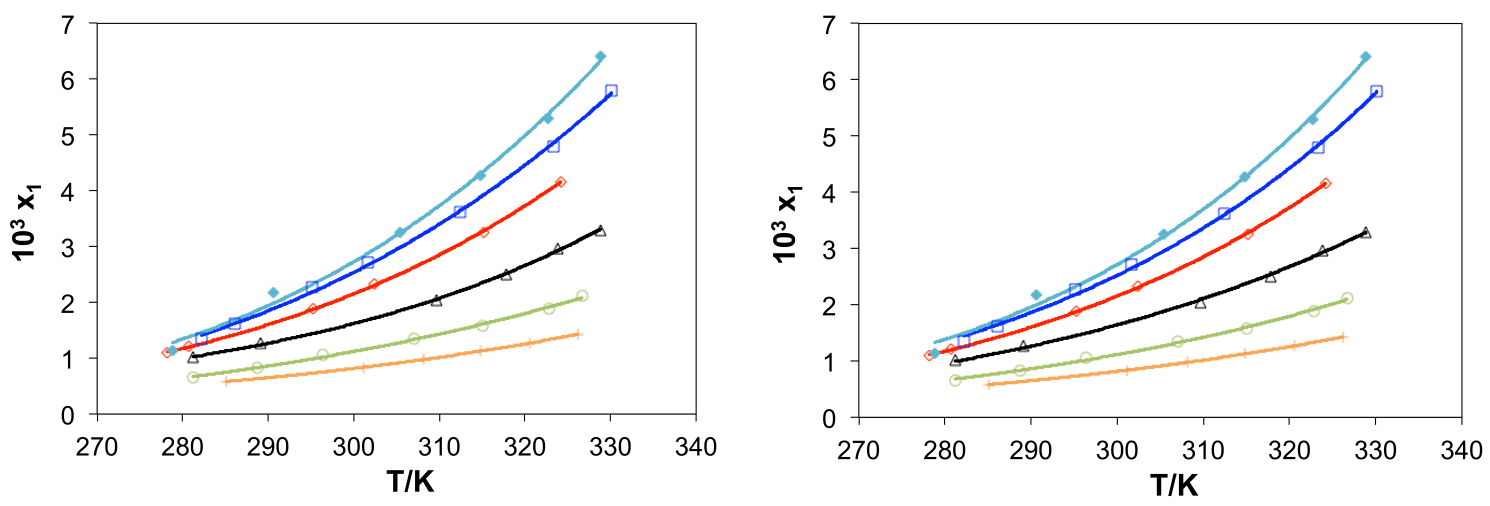

Figure S22. Experimental and correlated solubility data of WS - IPA (1) in ternary isopropanol (2) + water (3) with $W_{3}=0.01+$ heptane (4) solvent mixture. $\bullet, W_{4}=0$ (isopropanol + water $\left.W_{3}=0.01\right) ; \square, W_{4}=0.09 ; \diamond, W_{4}=0.18 ; \Delta, W_{4}=0.28 ; \circ, W_{4}=0.39 ;+, W_{4}=0.47 ;-$, calculated using the modified Apelblat equation (left) and $\lambda \mathrm{h}$ equation (right). $w_{3}$ is the mass fraction of water (3) in binary isopropanol (2) + water (3) mixture. $w_{4}$ is the mass fraction of heptane (4) in ternary isopropanol (2) + water (3) + heptane (4) mixture. $x_{1}$ represents the mole fraction solubility of WS • IPA and $T$ the temperature in kelvin $(\mathrm{K})$.

\section{Raman Spectroscopy}

Raman spectra were recorded at room temperature using a Raman Microscope (DXR2, Thermo Fisher Scientific) equipped with $532 \mathrm{~nm}$ laser, 400 lines/mm grating and $25 \mu \mathrm{m}$ slit. The spectra were collected over the range of $600-3400 \mathrm{~cm}^{-1}$ averaging 20 scans with $10 \mathrm{~s}$ exposure time per scan. Spectra obtained were analyzed using the OMNIC for Dispersive Raman software (version 9.2.0). Prior to the solubility measurements the commercial WS - IPA was analyzed by Raman microscopy and the solid-state was confirmed as the WS - IPA crystalline form. ${ }^{16}$ After the experiments were completed suspensions were measured by Raman microscopy to confirm that the yielded material was the WS - IPA crystalline form. For the ternary solvent system isopropanol + acetone + hexane solid material could only be recovered for the hexane mass fractions $\left(W_{4}\right)$ of 0.09 and 0.17 . It was demonstrated that the use of isopropanol as a solvent and co-solvent was necessary to obtain the desired crystalline material, WS - IPA. Using pure solvents and solvent mixtures containing acetone, ethanol, and water (without isopropanol) resulted in a gel-like WS (Figure 23). ${ }^{6,1718}$ These samples were not analyzed. Figures S24-S30 show representative Raman spectra for the WS • IPA crystals obtained. 
Figure S23. Representative picture of the resulting gel-like WS obtained when using pure solvents, in this case ethanol.

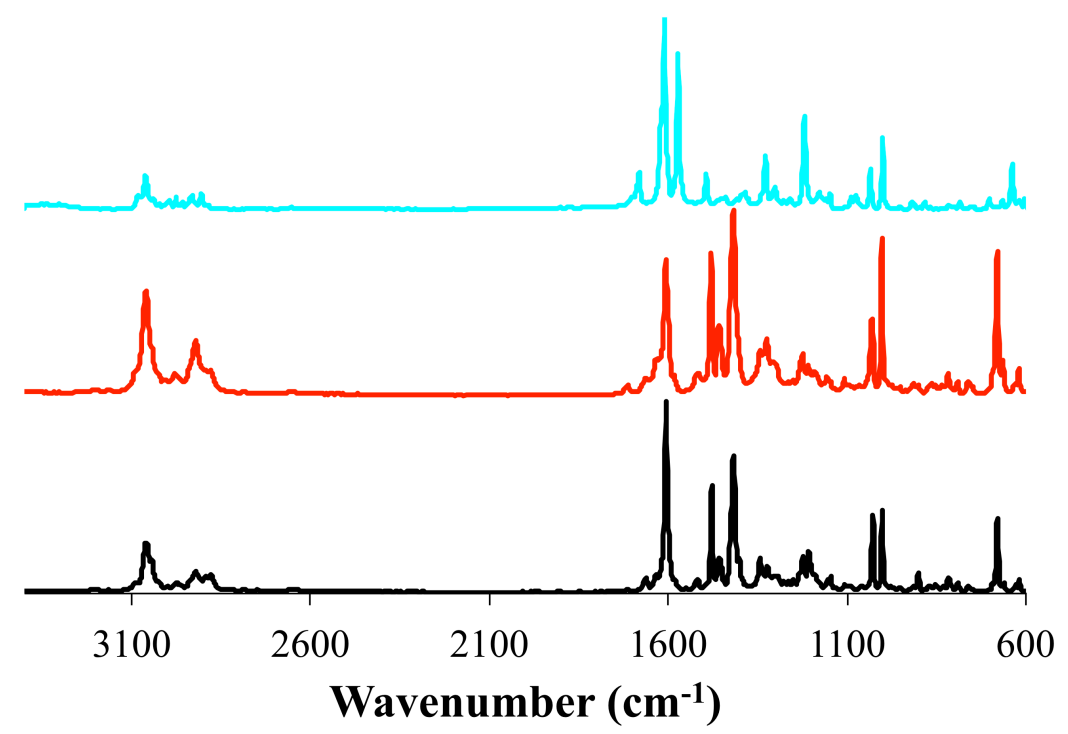

Figure S24. Representative Raman spectra of WS - IPA crystals obtained in isopropanol (red) and compared to commercial "as received" WS • IPA (black), and commercial "as received" Warfarin (cyan). 


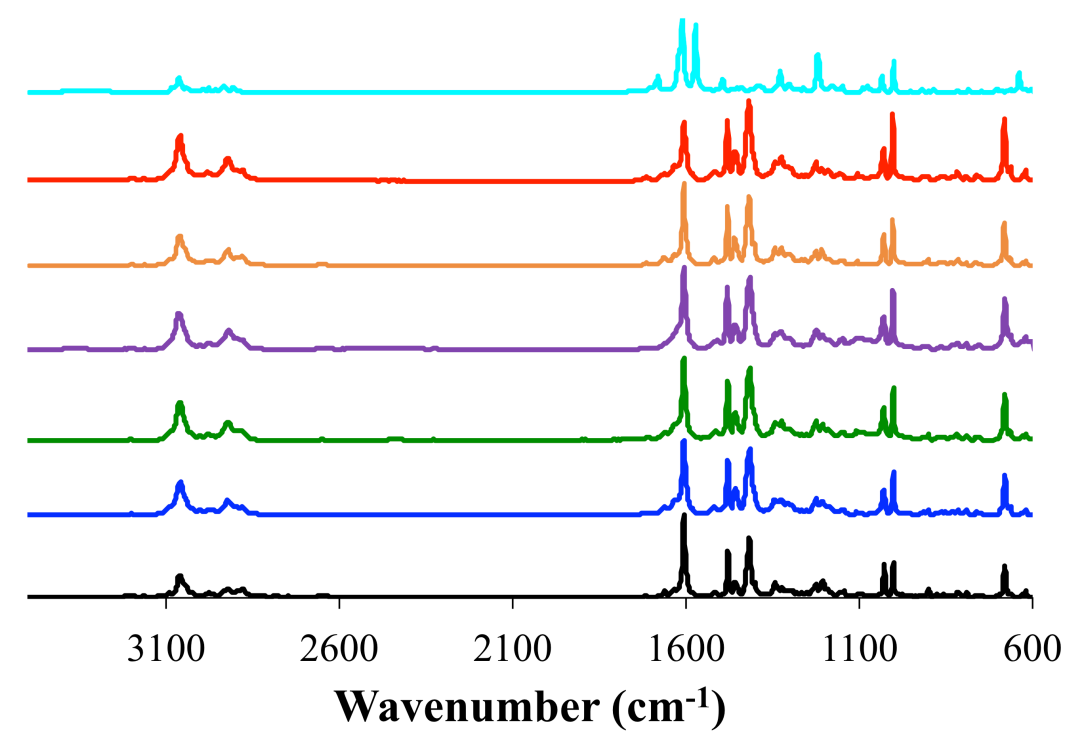

Figure S25. Representative Raman spectra of WS - IPA (1) crystals obtained in binary solvent systems (bottom to top); commercial "as received" WS - IPA (black), isopropanol + acetone (blue), isopropanol + ethanol (green), isopropanol + water (purple), isopropanol + heptane (orange), isopropanol + hexane (red), and commercial "as received" Warfarin (cyan).

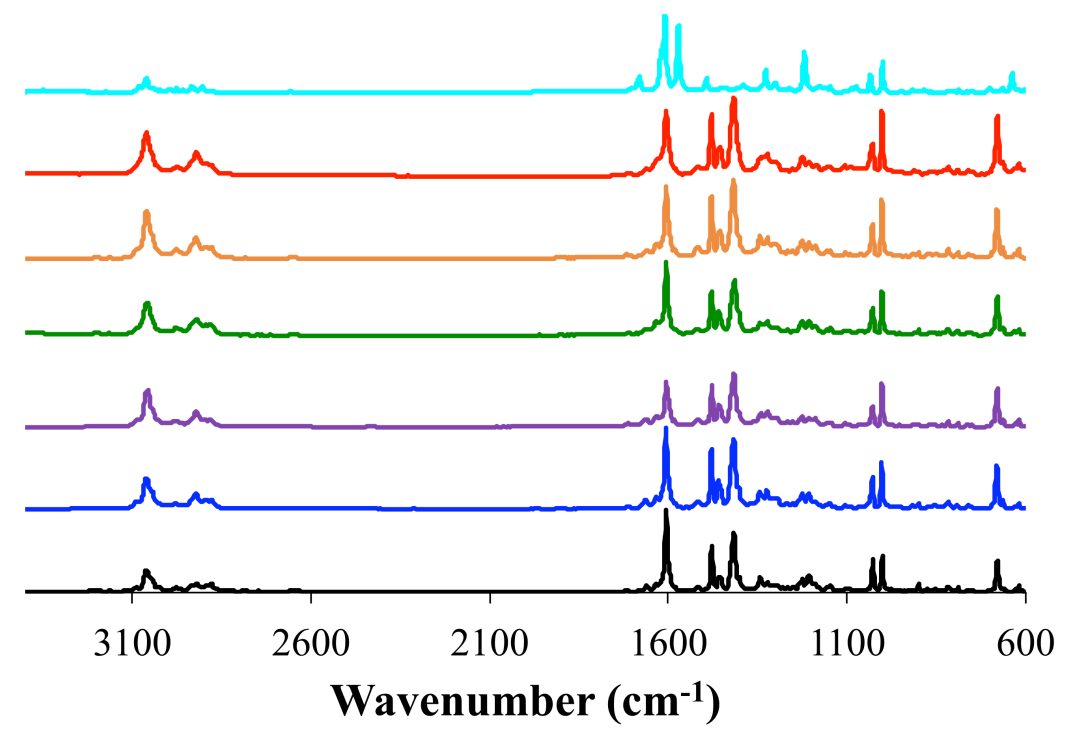

Figure S26. Representative Raman spectra of WS - IPA (1) crystals obtained in ternary isopropanol (2) + acetone (3) with $W_{3}=0.10+$ heptane (4) solvent system (bottom to top); commercial "as received" WS - IPA (black), $W_{4}=0.09$ (blue), $W_{4}=0.18$ (purple), $W_{4}=0.27$ (green), $W_{4}=0.37$ (orange), $W_{4}=0.47$ (red), and commercial "as received" Warfarin (cyan). $W_{3}$ is the mass fraction of acetone (3) in binary isopropanol (2) + acetone (3) solvent mixture. $W_{4}$ is the mass fraction of heptane (4) in the ternary isopropanol (2) + acetone (3) + heptane (4) solvent mixture. 


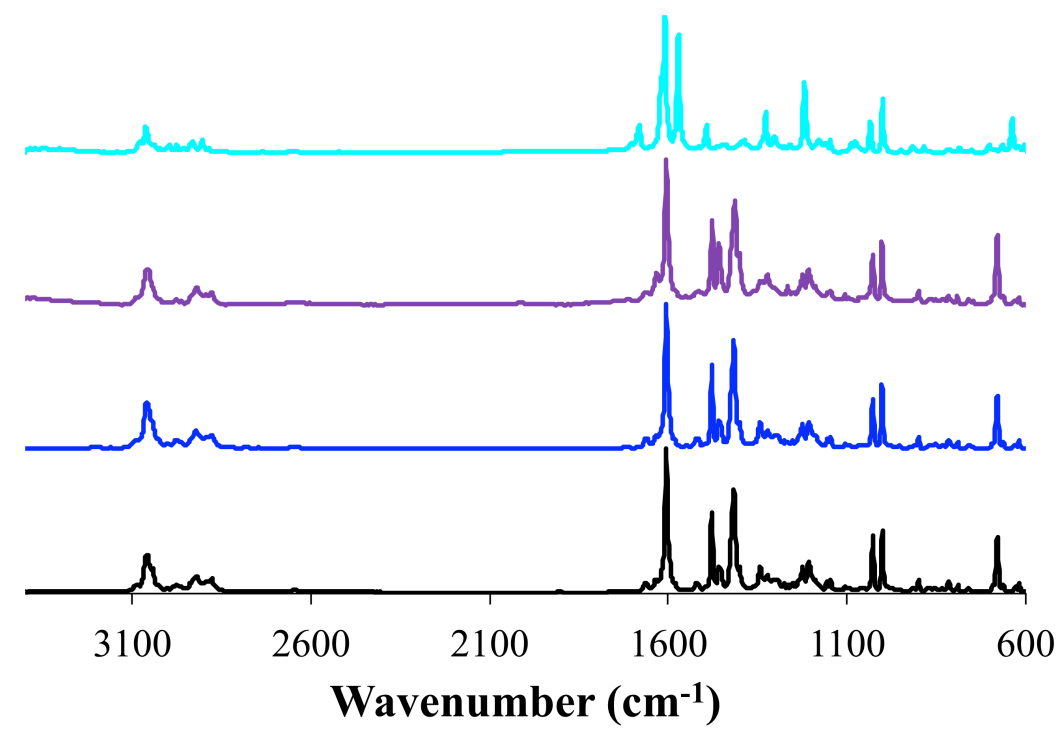

Figure S27. Representative Raman spectra of WS - IPA (1) crystals obtained in ternary isopropanol (2) + acetone (3) with $W_{3}=0.10+$ hexane (4) solvent system (bottom to top); commercial "as received" WS - IPA (black), $W_{4}=0.09$ (blue), $W_{4}=0.17$ (purple), and commercial "as received" Warfarin (cyan). $W_{3}$ is the mass fraction of acetone (3) in binary isopropanol (2) + acetone (3) solvent mixture. $W_{4}$ is the mass fraction of hexane (4) in the ternary isopropanol (2) + acetone (3) + hexane (4) solvent mixture.

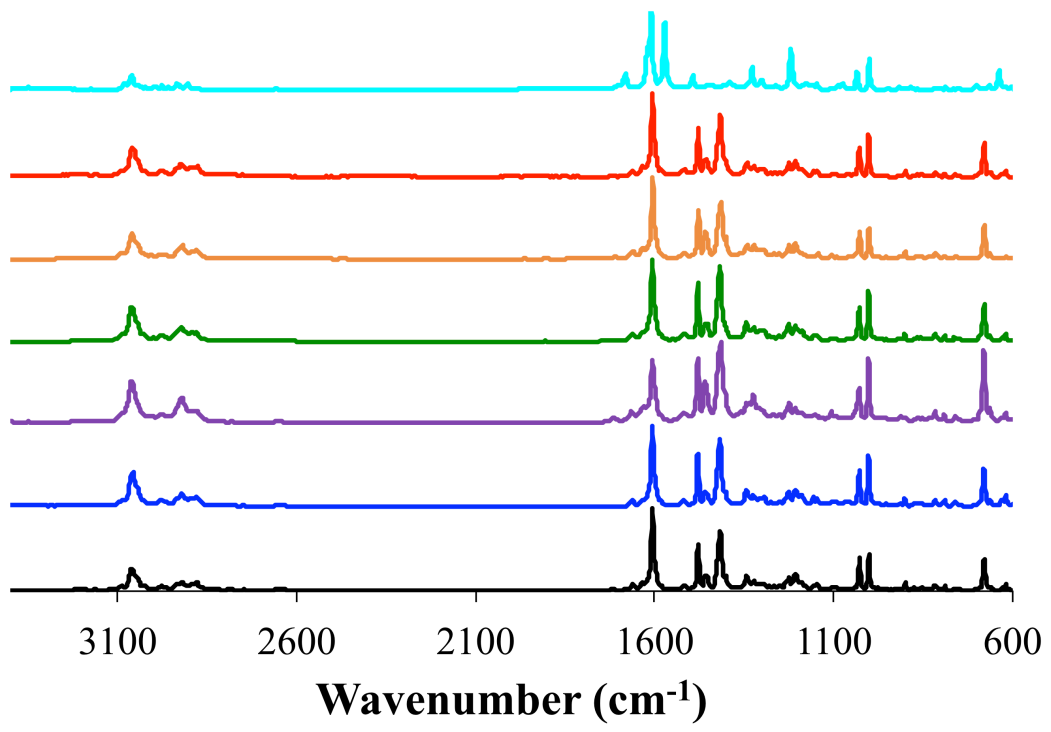

Figure S28. Representative Raman spectra of WS - IPA (1) crystals obtained in ternary isopropanol (2) + ethanol (3) with $W_{3}=0.20+$ heptane (4) solvent system (bottom to top); commercial "as received" WS - IPA (black), $W_{4}=0.09$ (blue), $W_{4}=0.18$ (purple), $W_{4}=0.27$ (green), $W_{4}=0.38$ (orange), $W_{4}=0.47$ (red), and commercial "as received" Warfarin (cyan). $W_{3}$ is the mass fraction of ethanol (3) in binary isopropanol (2) + ethanol (3) solvent mixture. $W_{4}$ is the mass fraction of heptane (4) in the ternary isopropanol (2) + ethanol (3) + heptane (4) solvent mixture. 


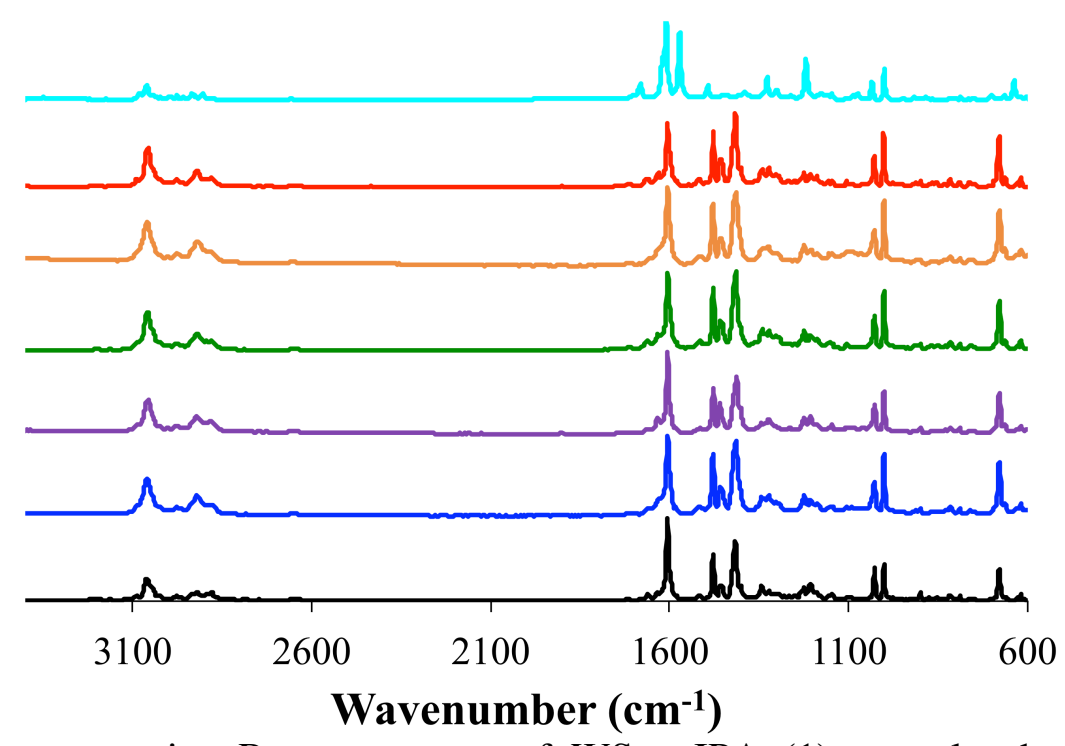

Figure S29. Representative Raman spectra of WS - IPA (1) crystals obtained in ternary isopropanol (2) + ethanol (3) with $W_{3}=0.20+$ hexane (4) solvent system (bottom to top); commercial "as received" WS - IPA (black), $W_{4}=0.08$ (blue), $W_{4}=0.17$ (green), $W_{4}=0.27$ (purple), $W_{4}=0.36$ (orange), $W_{4}=0.46$ (red), and commercial "as received" Warfarin (cyan). $W_{3}$ is the mass fraction of ethanol (3) in binary isopropanol (2) + ethanol (3) solvent mixture. $W_{4}$ is the mass fraction of hexane (4) in the ternary isopropanol (2) + ethanol (3) + hexane (4) solvent mixture.

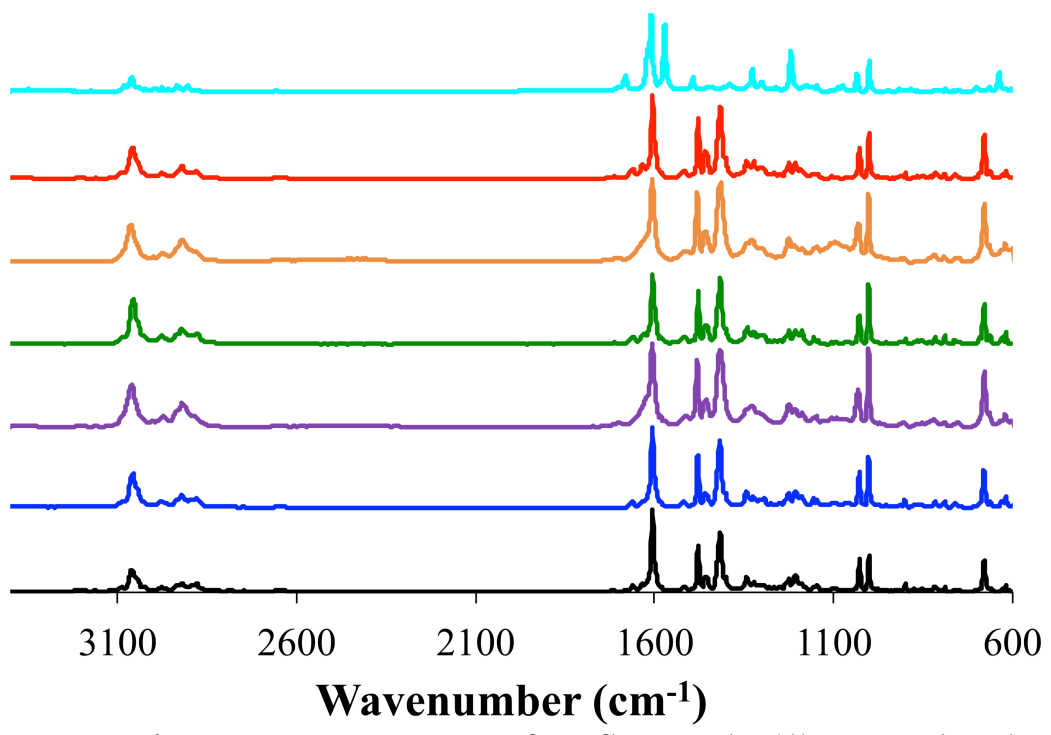

Figure S30. Representative Raman spectra of WS - IPA (1) crystals obtained in ternary isopropanol (2) + water (3) with $W_{3}=0.01+$ heptane (4) solvent system (bottom to top); commercial "as received" WS - IPA (black), $w_{4}=0.09$ (blue), $W_{4}=0.18$ (purple), $W_{4}=0.28$ (green), $W_{4}=0.39$ (orange), $W_{4}=0.47$ (red), and commercial "as received" Warfarin (cyan). $W_{3}$ is the mass fraction of water (3) in binary isopropanol (2) + water (3) solvent mixture. $W_{4}$ is the mass fraction of heptane (4) in the ternary isopropanol (2) + water (3) + heptane (4) solvent mixture. 


\section{Powder X-ray Diffraction (PXRD) Analysis}

Powder diffractograms were collected using a Rigaku XtaLAB SuperNova single microfocus $\mathrm{Cu}-\mathrm{K} \alpha$ radiation ( $\lambda=1.5417 \AA$ ) source equipped with a HyPix3000 X-ray detector in transmission mode operating at $50 \mathrm{kV}$ and $1 \mathrm{~mA}$. Powder samples were mounted in MiTeGen micro loops with a minimal amount of paratone oil. Powder diffractograms were collected at 300 $\mathrm{K}$ over an angular $2 \theta$ range between $7-40^{\circ}$ with a step of $0.01^{\circ}$ using the Gandolfi move experiment for powders (90 s exposure). Data were analyzed within the CrystAllis ${ }^{\text {rо }}$ software (v. 1.171.39.45c). Prior to the solubility measurements the commercial WS - IPA was analyzed by PXRD and the solid-state was confirmed as the WS - IPA crystalline form." After the experiments were completed suspensions were measured by PXRD and it was confirmed that the yielded material was the WS • IPA crystalline form (Figures S31-S37). For the ternary solvent system isopropanol + acetone + hexane solid material could only be recovered for the hexane mass fractions $\left(w_{4}\right)$ of 0.09 and 0.17. The PXRD data supports the findings of the Raman measurements (Figures S24-S30).

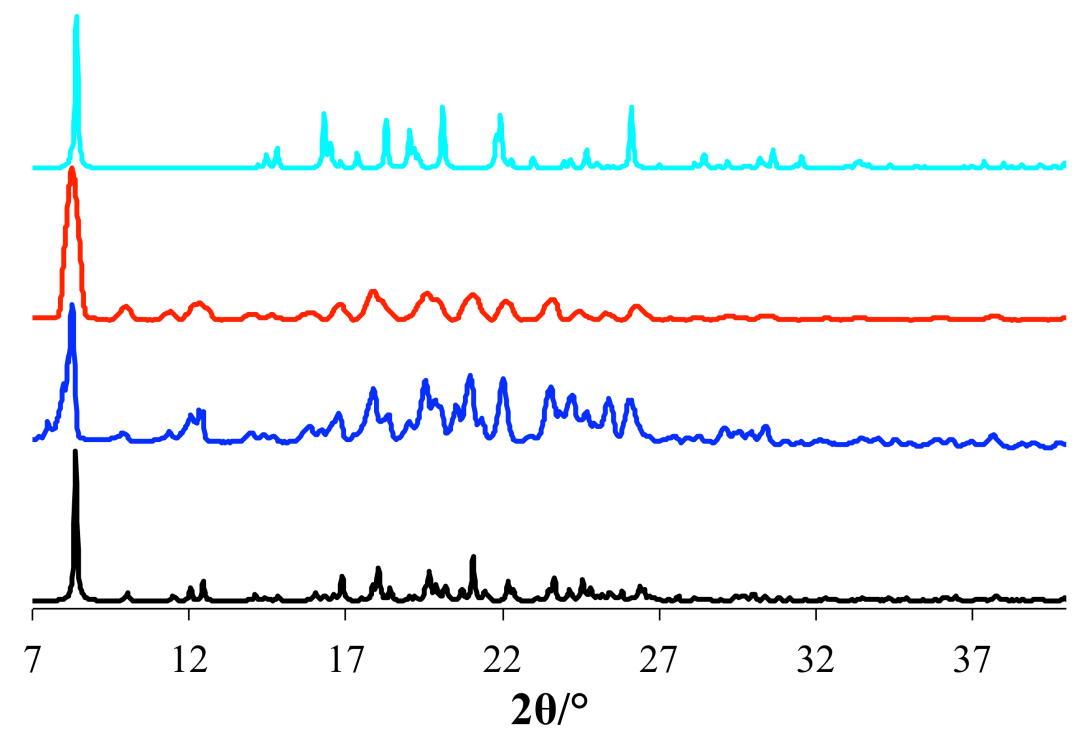

Figure S31. Representative powder X-ray diffractograms of WS - IPA recrystallized in isopropanol (red) compared to the simulated diffractogram of WS - IPA (Reference Code $=$ EFIWIZ") obtained from the Cambridge Structural Database, CSD (black), the "as received" WS IPA (blue), and the simulated diffractogram of warfarin (Reference Code $=$ BEFZES $^{20}$ ) obtained from the CSD (cyan). 


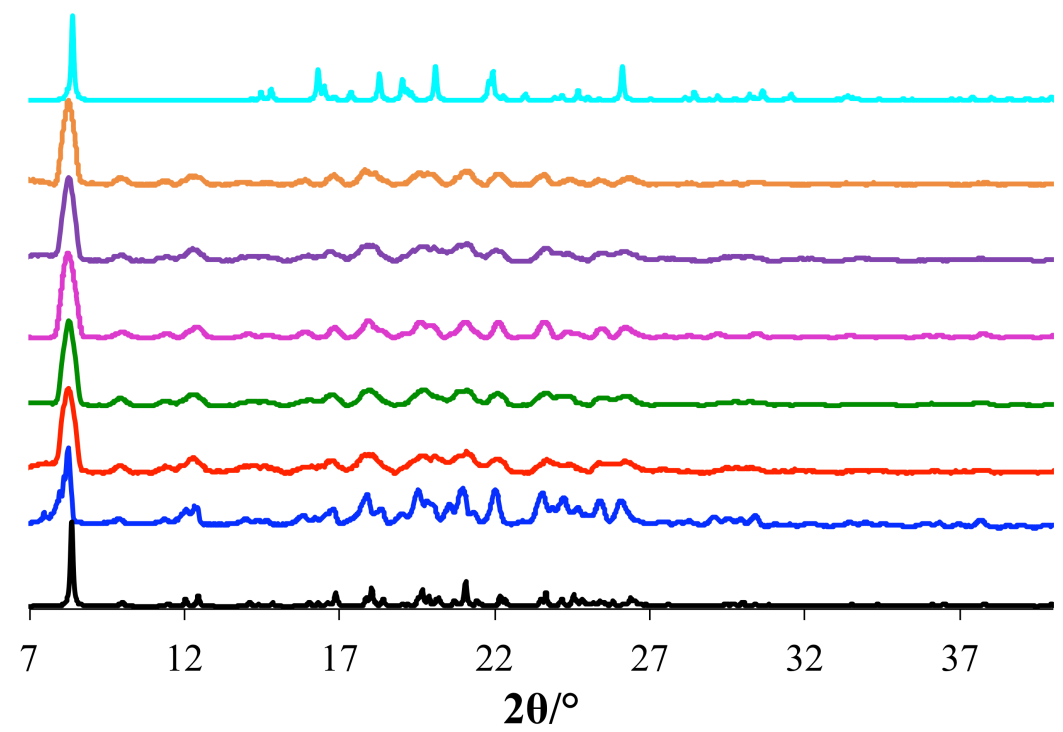

Figure S32. Representative powder X-ray diffractograms of WS - IPA crystals obtained in binary solvent systems; (bottom to top); the simulated diffractogram of WS - IPA (Reference Code $=$ EFIWIZ") obtained from the CSD (black), the "as received" WS - IPA white powder from vendor (blue), isopropanol + acetone (red), isopropanol + ethanol (green), isopropanol + water (magenta), isopropanol + heptane (purple), isopropanol + hexane (orange), and the simulated diffractogram of warfarin (Reference Code $=$ BEFZES $^{20}$ ) obtained from the CSD (cyan).

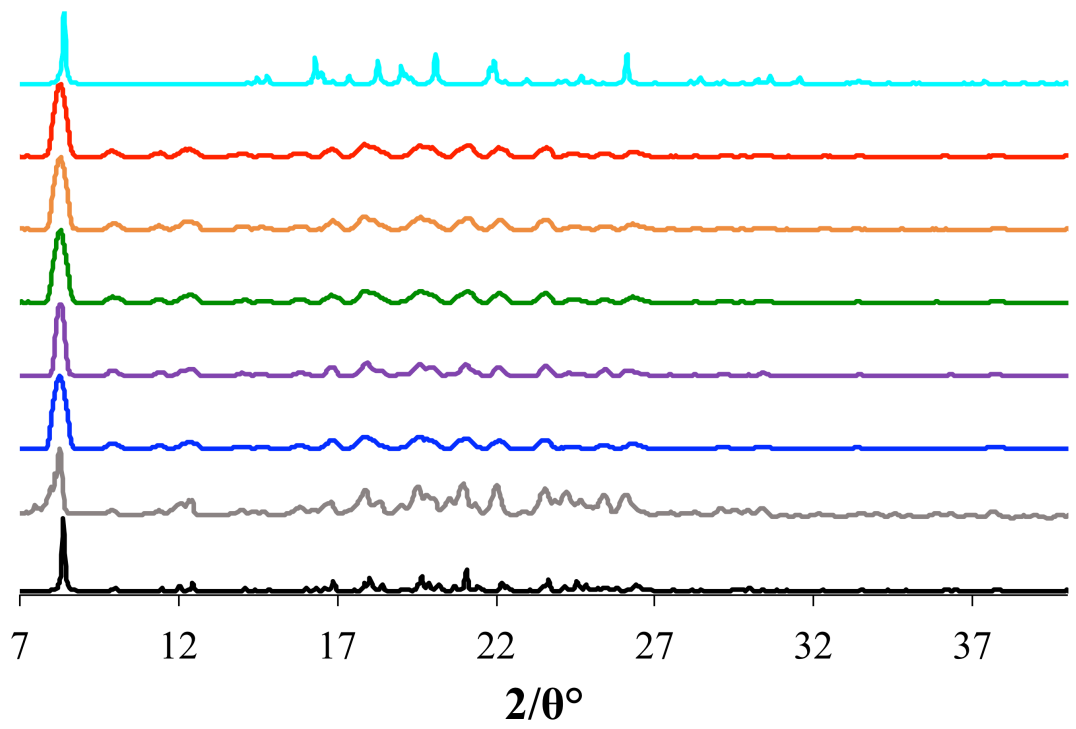

Figure S33. Representative powder X-ray diffractograms of WS - IPA (1) crystals obtained in ternary isopropanol (2) + acetone (3) with $W_{3}=0.10+$ heptane (4) solvent system (bottom to top); the simulated diffractogram of WS - IPA (Reference Code $=$ EFIWIZ ${ }^{\prime}$ ) obtained from the CSD (black), compared to the "as received" WS - IPA white powder from vendor (grey), $w_{4}=$ 0.09 (blue), $W_{4}=0.18$ (purple), $W_{4}=0.27$ (green), $W_{4}=0.37$ (orange), $W_{4}=0.47$ (red), and the simulated diffractogram of warfarin (Reference Code $=$ BEFZES $^{20}$ ) obtained from the CSD (cyan). $W_{4}$ is the mass fraction of heptane (4) in the ternary isopropanol (2) + acetone (3) + heptane (4) solvent mixture. 


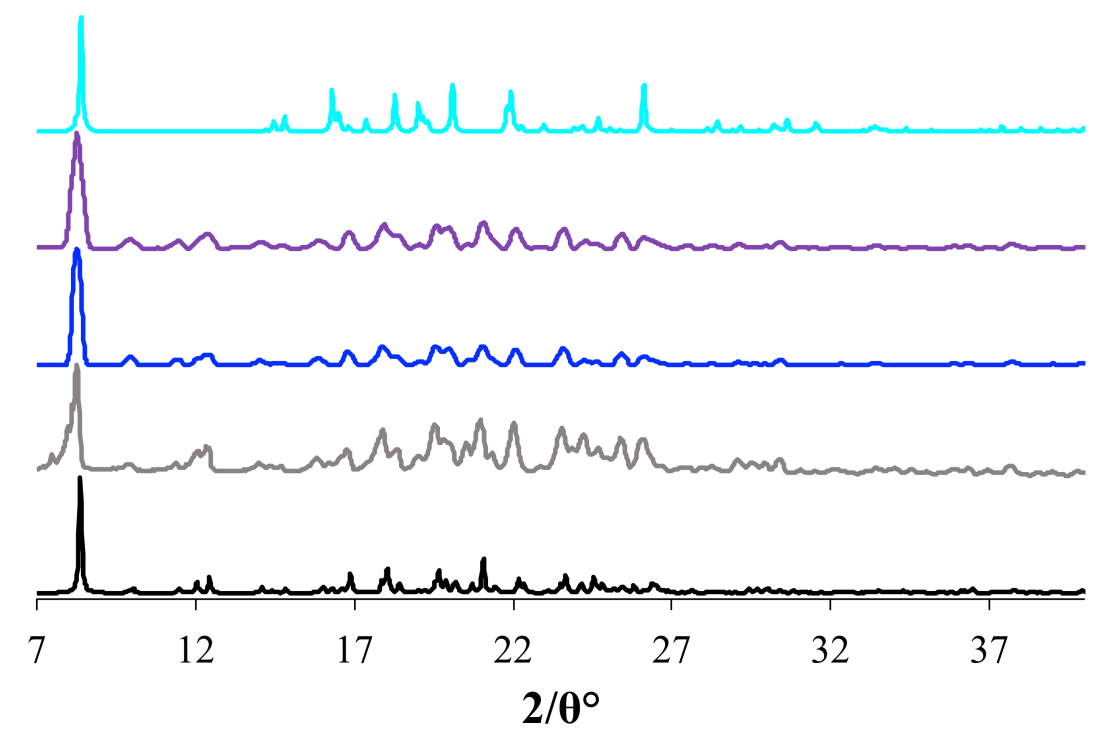

Figure S34. Representative powder X-ray diffractograms of WS - IPA (1) crystals obtained in ternary isopropanol (2) + acetone (3) with $W_{3}=0.10+$ hexane (4) solvent system (bottom to top); the simulated diffractogram of WS - IPA (Reference Code $=$ EFIWIZ ${ }^{\circ}$ ) obtained from the CSD (black), compared to the "as received" WS - IPA white powder from vendor (grey), $W_{4}=0.09$ (blue), $W_{4}=0.17$ (purple), and the simulated diffractogram of warfarin (Reference Code $=$ BEFZES ${ }^{\star}$ ) obtained from the CSD (cyan). $W_{4}$ is the mass fraction of hexane (4) in the ternary isopropanol (2) + acetone (3) + hexane (4) solvent mixture.

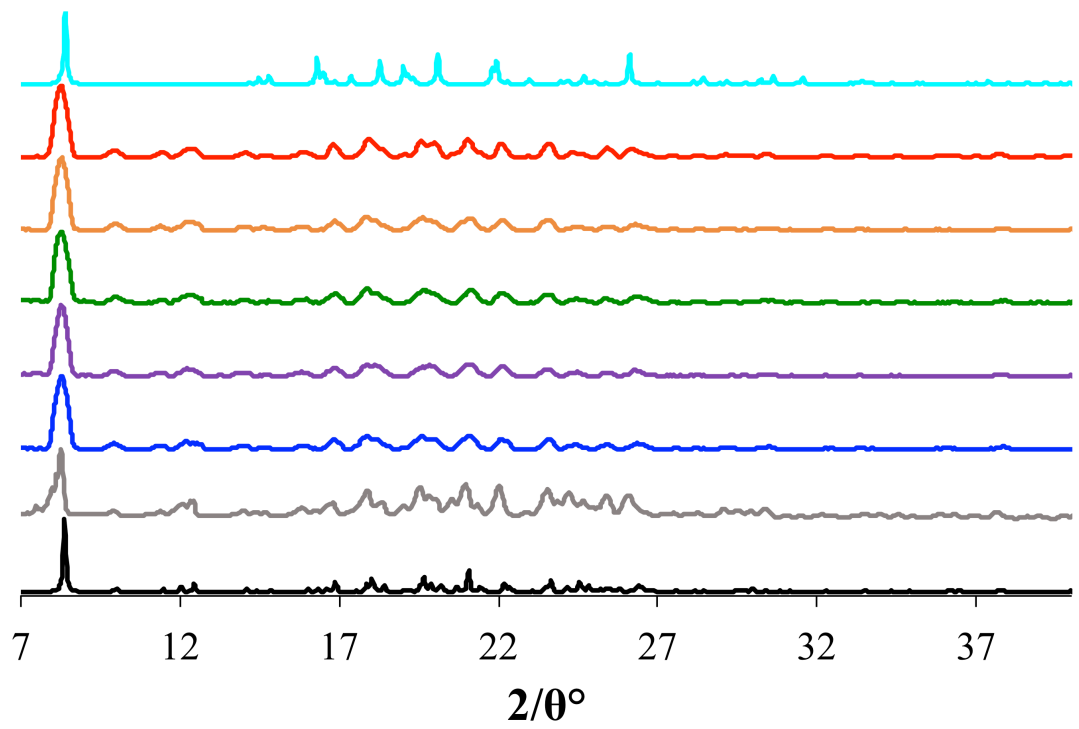

Figure S35. Representative powder X-ray diffractograms of WS - IPA (1) crystals obtained in ternary isopropanol (2) + ethanol (3) with $W_{3}=0.20+$ heptane (4) solvent system (bottom to top); the simulated diffractogram of WS - IPA (Reference Code $=$ EFIWIZ" ) obtained from the CSD (black), compared to the "as received" WS - IPA white powder from vendor (grey), $W_{4}=0.09$ (blue), $W_{4}=0.18$ (purple), $W_{4}=0.27$ (green), $W_{4}=0.38$ (orange), $W_{4}=0.47$ (red), and the simulated diffractogram of warfarin (Reference Code $=$ BEFZES $^{*}$ ) obtained from the CSD (cyan). $W_{4}$ is the mass fraction of heptane (4) in the ternary isopropanol (2) + ethanol (3) + heptane (4) solvent mixture. 


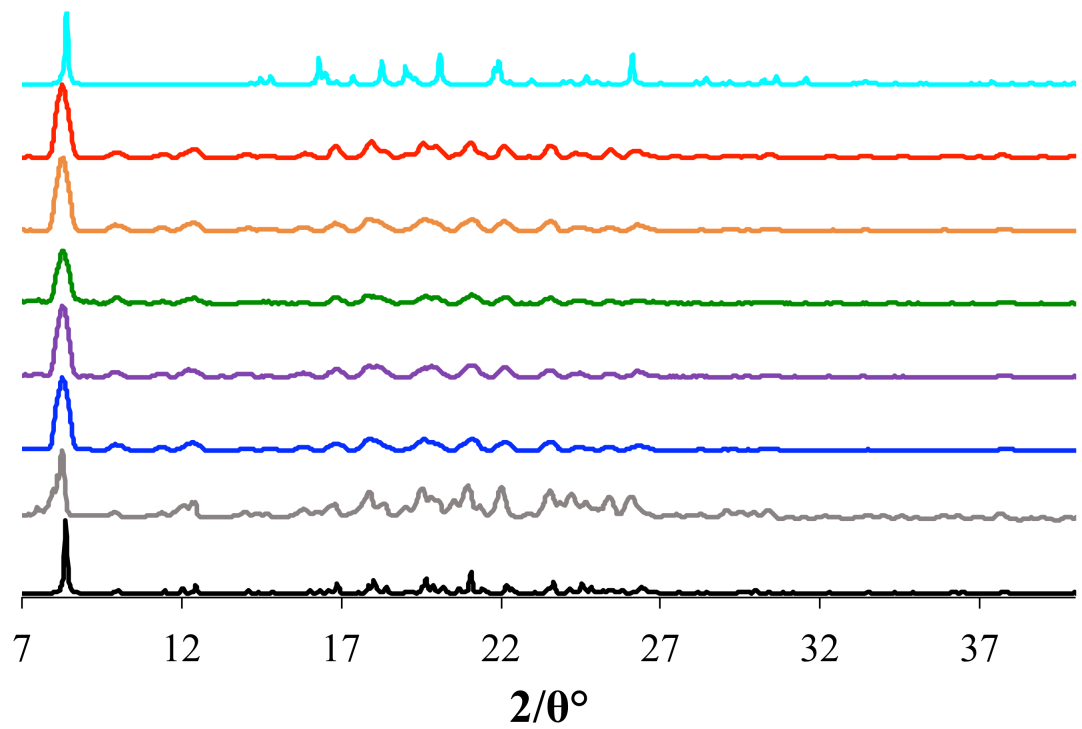

Figure S36. Representative powder X-ray diffractograms of WS - IPA (1) crystals obtained in ternary isopropanol (2) + ethanol (3) with $W_{3}=0.20+$ hexane (4) solvent system (bottom to top); the simulated diffractogram of WS $\bullet$ IPA (Reference Code $=$ EFIWIZ ${ }^{19}$ ) obtained from the CSD (black), compared to the "as received" WS • IPA white powder from vendor (grey), $W_{4}=0.08$ (blue), $W_{4}=0.17$ (purple), $W_{4}=0.27$ (green), $W_{4}=0.36$ (orange), $W_{4}=0.46$ (red), and the simulated diffractogram of warfarin (Reference Code $=$ BEFZES $^{20}$ ) obtained from the CSD (cyan). $W_{4}$ is the mass fraction of hexane (4) in the ternary isopropanol (2) + ethanol (3) + hexane (4) solvent mixture.

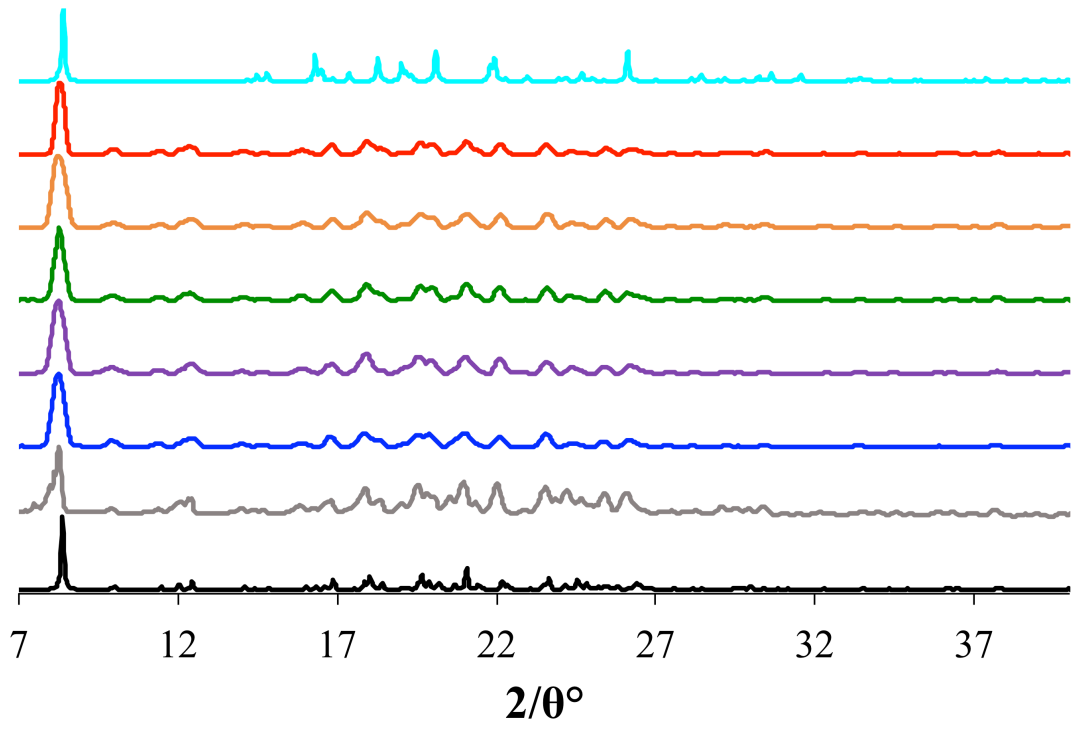

Figure S37. Representative powder X-ray diffractograms of WS - IPA (1) crystals obtained in ternary isopropanol (2) + water (3) with $W_{3}=0.01+$ heptane (4) solvent system (bottom to top); the simulated diffractogram of WS $\bullet$ IPA $\left(\right.$ Reference Code $=$ EFIWIZ $\left.^{1}\right)$ obtained from the CSD (black), compared to the "as received" WS - IPA white powder from vendor (grey), $W_{4}=0.09$ (blue), $W_{4}=0.18$ (purple), $W_{4}=0.28$ (green), $W_{4}=0.39$ (orange), $W_{4}=0.47$ (red), and the simulated diffractogram of warfarin (Reference Code $=$ BEFZES $^{20}$ ) obtained from the CSD (cyan). $W_{4}$ is the mass fraction of heptane (4) in the ternary isopropanol (2) + water (3) + heptane (4) solvent mixture. 


\section{References}

(1) Ahuja, S.; Scypinski, S. Handbook of Modern Pharmaceutical Analysis, 2nd ed.; Vol. 10, Academic Press, Amsterdam, 2011.

(2) Brittain, H. G. Polymorphism in Pharmaceutical Solids, 2nd ed.; Informa Healthcare, USA, Inc.: New York, 2009.

(3) Tian, Y.; Booth, J.; Meehan, E.; Jones, D. S.; Li, S.; Andrews, G. P. Construction of Drug-Polymer Thermodynamic Phase Diagrams Using Flory-Huggins Interaction Theory: Identifying the Relevance of Temperature and Drug Weight Fraction to Phase Separation within Solid Dispersions. Mol. Pharm. 2013, 10, 236-248.

(4) Giron, D. Thermal Analysis and Calorimetric Methods in the Characterisation of Polymorphs and Solvates. Thermochim. Acta 1995, 248, 1-59.

(5) Weldele, M.; Delmarre, D.; Gao, D.; Assad El-Khateeb, M.; Sison Centeno, C.-J.; Ratnakar Pathak, S. Stable Warfarin Sodium Liquid Formulation and Method of Making Same. U.S. Pat. 7,259,185, 2007.

(6) Gao, D.; Maurin, M. B. Physical Chemical Stability of Warfarin Sodium. AAPS PharmSci 2001, 3, E3.

(7) Ahuja, S.; Scypinski, S. Handbook of Modern Pharmaceutical Analysis; Academic Press, 2001.

(8) Nyvlt; Nývlt, J. Kinetics of Nucleation in Solutions. J. Cryst. Growth 1968, 3, 377-383.

(9) Kaemmerer, H.; Jones, M. J.; Lorenz, H.; Seidel-Morgenstern, A. Selective Crystallisation of a Chiral Compound-Forming System-Solvent Screening, SLE Determination and Process Design. Fluid Phase Equilib. 2010, 296, 192-205.

(10) Vellema, J.; Hunfeld, N. G. M.; Van Den Akker, H. E. A.; Ter Horst, J. H. Avoiding Crystallization of Lorazepam during Infusion. Eur. J. Pharm. Sci. 2011, 44, 621-626.

(11) Wei, T.; Wang, C.; Du, S.; Wu, S.; Li, J.; Gong, J. Measurement and Correlation of the Solubility of Penicillin V Potassium in Ethanol + Water and 1-Butyl Alcohol + Water Systems. J. Chem. Eng. Data 2015, 60,112-117. 
(12) Reus, M. A.; Van Der Heijden, A. E. D. M.; Ter Horst, J. H. Solubility Determination from Clear Points upon Solvent Addition. Org. Process Res. Dev. 2015, 19, 1004-1011.

(13) Monbaliu, J.-C. M.; Stelzer, T.; Revalor, E.; Weeranoppanant, N.; Jensen, K. F.; Myerson, A. S. Compact and Integrated Approach for Advanced End-to-End Production, Purification, and Aqueous Formulation of Lidocaine Hydrochloride. Org. Process Res. Dev. $2016,20,1347-1353$.

(14) Zorrilla-Veloz, R. I.; Stelzer, T.; López-Mejías, V. Measurement and Correlation of the Solubility of 5-Fluorouracil in Pure and Binary Solvents. J. Chem. Eng. Data 2018, 63, 38093817.

(15) Smallwood, I. M. Handbook of Organic Solvent Properties; Arnold: London, Great Britain, 1996.

(16) Rahman, Z.; Mohammad, A.; Akhtar, S.; Siddiqui, A.; Korang-Yeboah, M.; Khan, M. A. Chemometric Model Development and Comparison of Raman And13C Solid-State Nuclear Magnetic Resonance-Chemometric Methods for Quantification of Crystalline/Amorphous Warfarin Sodium Fraction in the Formulations. J. Pharm. Sci. 2015, 104, 2550-2558.

(17) Hiskey, C. F.; Melnitchenko, V. Clathrates of Sodium Warfarin. J. Pharm. Sci. 1965, 54 (9), 1298-1302.

(18) Rahman, Z.; Korang-Yeboah, M.; Siddiqui, A.; Mohammad, A.; Khan, M. A. Understanding Effect of Formulation and Manufacturing Variables on the Critical Quality Attributes of Warfarin Sodium Product. Int. J. Pharm. 2015, 495, 19-30.

(19) Sheth, A. R.; Brennessel, W. W.; Young, V. G.; Muller, F. X.; Grant, D. J. W. Solid-State Properties of Warfarin Sodium 2-Propanol Solvate. J. Pharm. Sci. 2004, 93, 2669-2680.

(20) Halland, N.; Hansen, T.; Jørgensen, K. A. Organocatalytic Asymmetric Michael Reaction of Cyclic 1,3-Dicarbonyl Compounds And $\alpha, \beta$-Unsaturated Ketones-A Highly Atom-Economic Catalytic One-Step Formation of Optically Active Warfarin Anticoagulant. Angew. Chemie Int. Ed. 2003, 42, 4955-4957. 